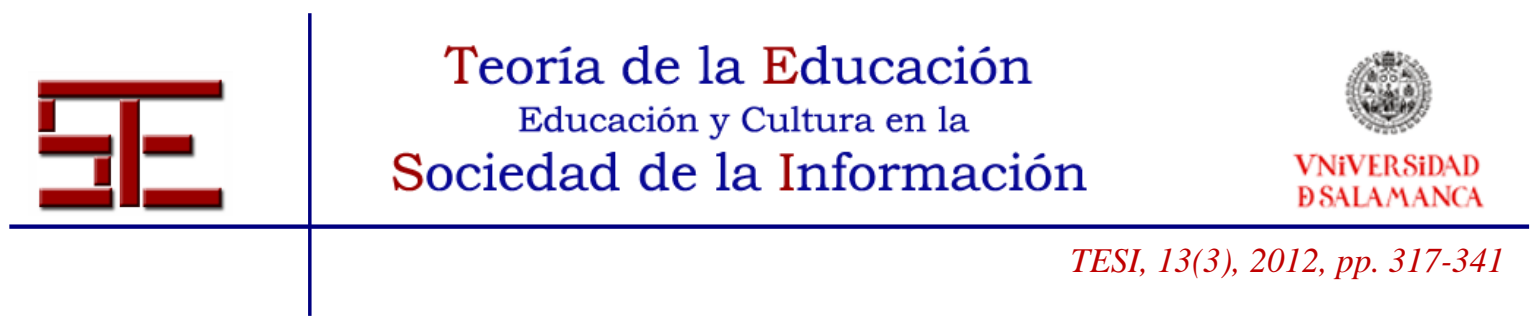

\title{
PERCEPCIÓN DE LOS FUTUROS DOCENTES SOBRE LOS SISTEMAS DE EVALUACIÓN DE SUS APRENDIZAJES ${ }^{1}$
}

Resumen: La finalidad de este estudio es analizar la percepción que el alumnado tiene de los sistemas, instrumentos y técnicas de evaluación y calificación que se utilizan en la formación inicial del profesorado (FIP). Para determinar la situación actual de esta evaluación en la FIP se ha elaborado un cuestionario con cuatro subescalas que ha presentado un nivel alto de fiabilidad. Los datos obtenidos, tras su aplicación a una muestra de 635 alumnos, pertenecientes a 7 centros universitarios, se analizan tanto a nivel descriptivo como inferencial utilizando ANOVAs de un factor. Los resultados indican que las prácticas de evaluación orientadas al aprendizaje son todavía minoritarias en la FIP. En cambio es preocupante la existencia de numerosas diferencias significativas entre la FIP de primaria y de secundaria. Los análisis sugieren, además, que existen pocas asignaturas con estilos de evaluación orientados al aprendizaje, pero en ellas se utilizan estrategias muy variadas y ricas.

Palabras clave: Enseñanza Superior, Evaluación Formativa, Evaluación Orientada al Aprendizaje, Formación del Profesorado.

\footnotetext{
${ }^{1}$ Agradecimientos

Este artículo forma parte de la ejecución del proyecto de investigación titulado: “La docencia universitaria ante el Espacio Europeo de Educación Superior (EEES). Análisis de la situación actual en la Formación Inicial del Profesorado y desarrollo de propuestas basadas en el sistema ECTS y el desarrollo de competencias profesionales", subvencionado por la Junta de Castilla y León, en la convocatoria pública para el desarrollo de proyectos de investigación a iniciar en el 2008.
}

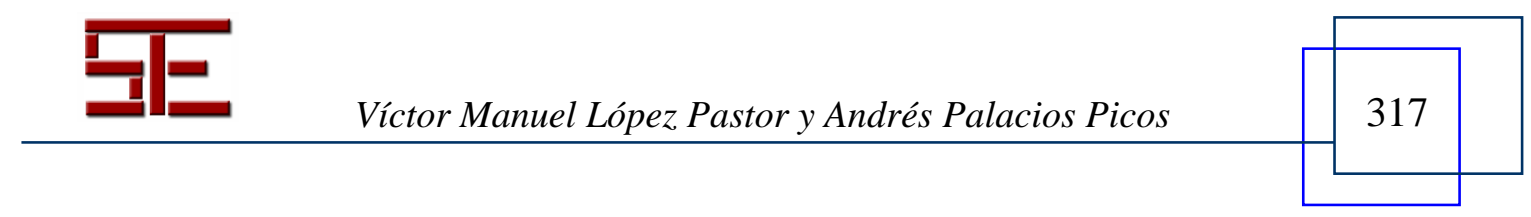




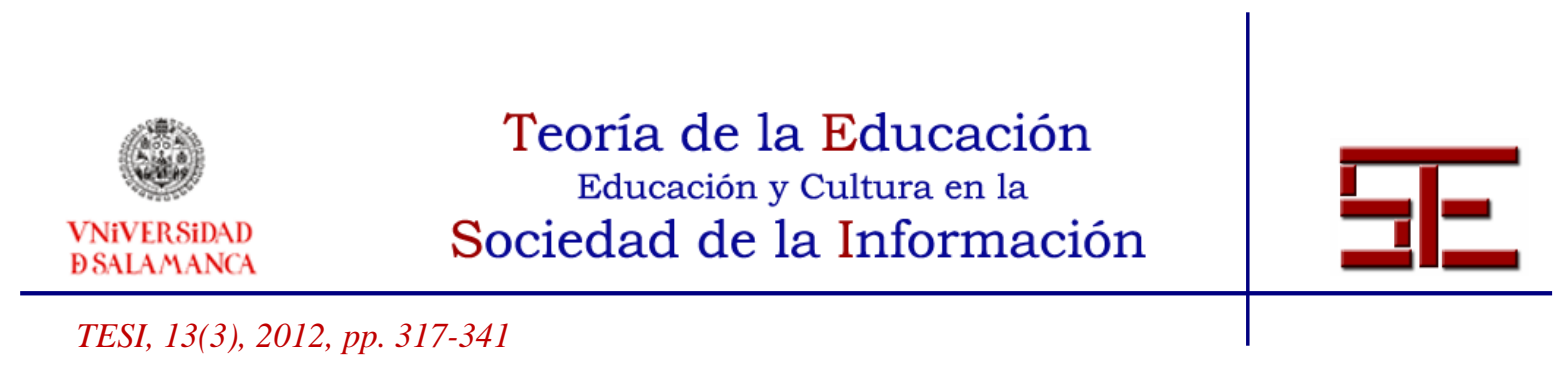

\title{
FUTURE TEACHERS' PERCEPTION ON THE ASSESSING SYSTEMS FOR THEIR LEARNING
}

\begin{abstract}
This paper is aimed at analysing the students' perception on assessment and grading systems, instruments and techniques used in Teacher Education (TE). In order to determine the current situation for this assessment in TE a questionnaire containing four subscales was developed with a high reliability degree. Data obtained are applied to a sample of 635 students from 7 different colleges and analysed with both descriptive and inferential statistics using single-factor ANOVA. The outcomes show that assessing practices oriented towards learning are still minority practices in TE. On the other hand, the existence of so many significant differences between Primary Teacher Education and Secondary Teacher Education is worrisome. The analyses also suggest there are few subjects containing assessing styles oriented towards learning, although they include very varied and rich strategies.
\end{abstract}

Keywords: Higher Education; Formative Assessment; Learing-oriented Assessment; Teacher Education.

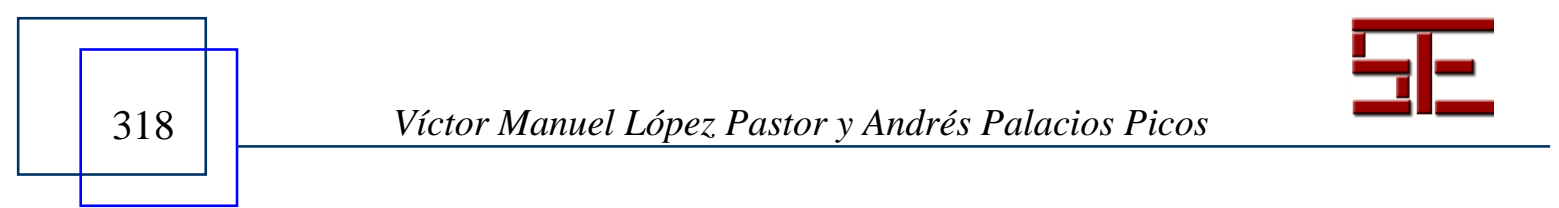




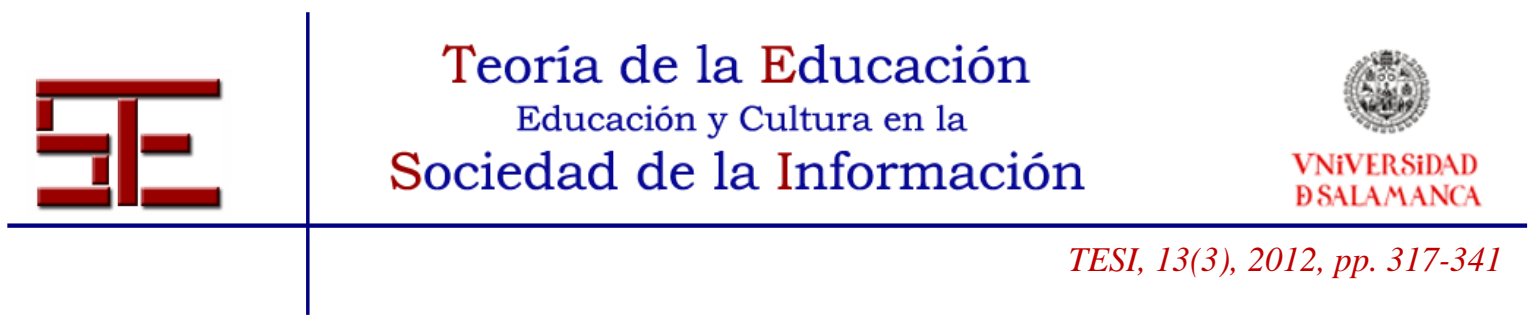

\section{PERCEPCIÓN DE LOS FUTUROS DOCENTES SOBRE LOS SISTEMAS DE EVALUACIÓN DE SUS APRENDIZAJES}

Fecha de recepción: 24/09/2011; fecha de aceptación: 18/09/2012; fecha de publicación: 30/11/2012

Víctor Manuel López Pastor

vlopez@mpc.uva.es

E.U. Magisterio de Segovia. Universidad de Valladolid

Andrés Palacios Picos

palacios@psi.uva.es

E.U. Magisterio de Segovia. Universidad de Valladolid

\section{1.- INTRODUCCIÓN}

Es notoria la existencia en la literatura especializada de dos enfoques opuestos en la enseñanza universitaria a la hora de plantear la evaluación del alumnado: una Evaluación Orientada a la Calificación (única o predominantemente sumativa y final) vs. una Evaluación Orientada al Aprendizaje (predominantemente formativa y continua). El concepto de Evaluación Orientada al Aprendizaje (EOA) es utilizado por numerosos autores en los últimos años (Brown \& Glasner, 2003; Carless, Joughin y Mok, 2006; Boud y Falchikov, 2007; Ibarra y Rodriguez-Gómez, 2010). Dicho concepto pone el énfasis en la utilización de la evaluación para generar un mayor aprendizaje en el alumnado. Brockbank y McGill (2002) explican que el término assessment proviene de una raíz latina cuyo significado es sentarse al lado de, en el sentido de dar una ayuda o cooperar con, en vez del significado de inspección y control, que es la forma habitual de entender y practicar la evaluación en educación superior. Ibarra y Rodríguez-Gómez (2010) consideran que este enfoque se fundamenta en tres aspectos: (a)-tareas de evaluación auténticas y ajustadas a los propósitos; (b)implicación de los estudiantes en el proceso de evaluación; (c)-retroalimentación con perspectiva de futuro y posibilidades de mejoras reales.

El enfoque de evaluación orientada al aprendizaje ha sido considerado desde diferentes puntos de vista como una pieza clave de mejora docente. Así, Dochy, Segers y Dierick (2002) consideran que el reto más importante de nuestras universidades es pasar de una cultura del examen a una cultura de la evaluación, entendiendo por esta última una evaluación formativa, dirigida a mejorar el proceso de aprendizaje del alumnado y a

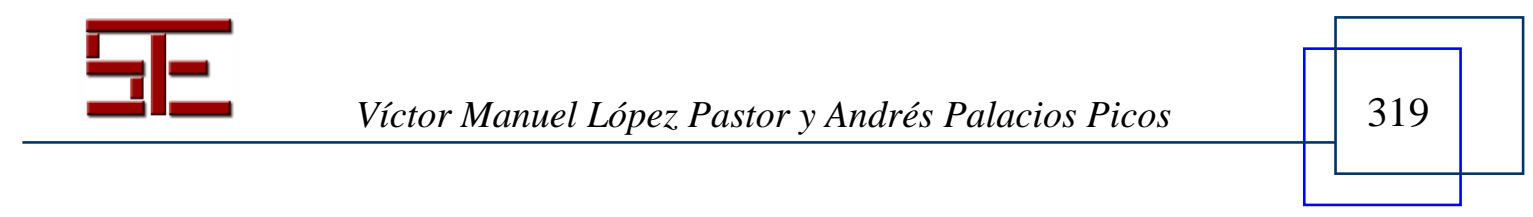




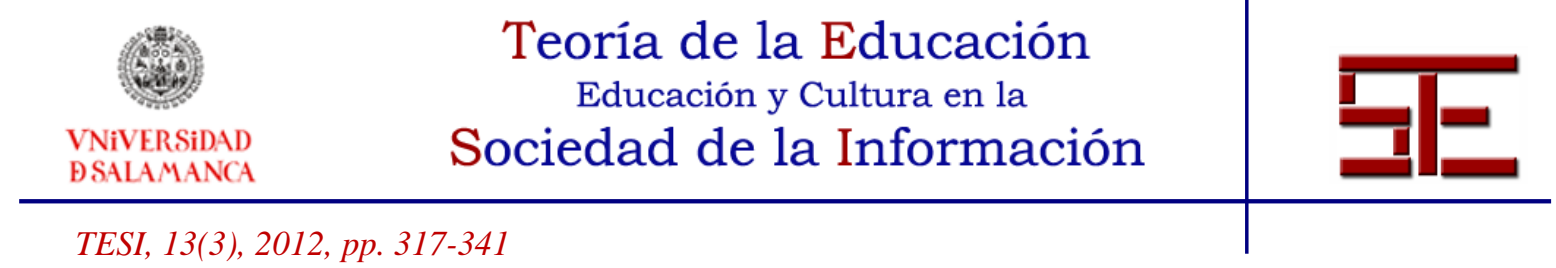

favorecer su participación en los procesos evaluativos. Esta idea es también recogida por Sanmartí (2007), para quien hay que implicar al alumnado en el proceso de evaluación fomentando la capacidad de realizar, con criterios y conocimiento, la evaluación de su propio proceso de aprendizaje, así como el de sus compañeros de trabajo. Shepard (2000) señala que si queremos que la evaluación tenga la finalidad de ayudar a los estudiantes a aprender, debe modificarse de dos formas fundamentales: (a)mejorar el contenido y el carácter de las evaluaciones; (b)-hacer que la información obtenida en la evaluación forme parte del proceso de aprendizaje.

Boud y Falchikov (2007) defienden que en la educación superior habría que dar más énfasis a la evaluación orientada al aprendizaje a largo plazo, para que los estudiantes puedan enfrentarse a tipos de aprendizajes muy contextualizados, propios de la vida y el trabajo. También defienden el desarrollo de una evaluación alternativa que prime la implicación del estudiante, a través de tareas auténticas que permitan una retroalimentación eficaz con posibilidad de cambio y mejora. Ideas que comparte Knight (2005), quien afirma que la evaluación formativa tiene un gran potencial para mejorar el aprendizaje, dado que se ha demostrado que las buenas prácticas de evaluación formativa pueden conducir a unos progresos en el aprendizaje mayores que cualquier otra innovación educativa. Estas ideas se basan en los trabajos anteriores de Black y William (1998), quienes, tras la revisión de 681 artículos de investigación sobre evaluación formativa, demuestran de manera concluyente que la evaluación formativa mejora el aprendizaje y que el posible efecto de mejora está entre los mayores con respecto a intervenciones educativas.

En todos estos trabajos, se subraya la idea del importante papel dinamizador que tiene la evaluación, ya que condiciona poderosamente los procesos de aprendizaje del alumnado, así como sus estrategias y esfuerzos (Biggs, 2005; Gibbs, 2003; Sanmartí, 2007; Zabalza, 2003), por lo que ninguna innovación didáctica será efectiva si no va acompañada de innovaciones en el modo de concebir la evaluación (Bonsón y Benito, 2005; Dochy, Segers, Dierik, 2002).

No obstante, los estudios sobre el tipo de evaluación que predomina en las aulas universitarias parecen indicar que se han producido escasos cambios en los últimos años y que el uso de procesos de evaluación formativa y continua sigue siendo poco habitual (Fernández-Pérez, 1989; Flórez, 1999; Nieto, 2000; Rodríguez-Gómez et al., 1998; Tejedor, 1998; Trillo, 2005; Trillo y Porto, 1999; Zabalza, 2003). En la realidad de estas aulas, el concepto evaluación no se suele interpretar en su sentido más amplio y abierto

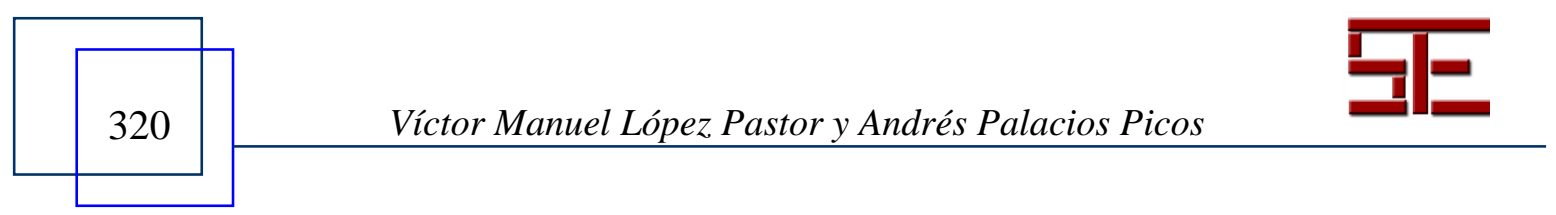




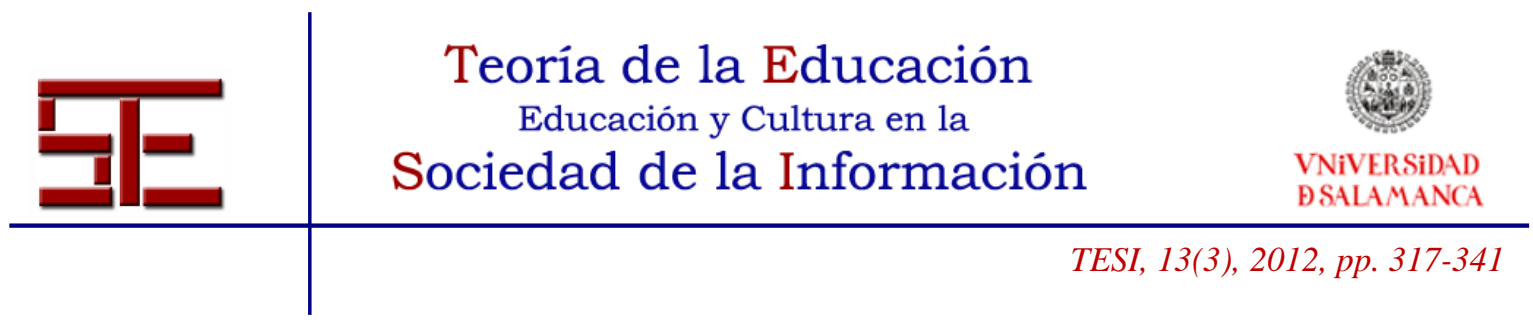

que acabamos de comentar, sino como un mero sinónimo reduccionista de calificación, o cualquiera de los diferentes términos integrados en su campo semántico: nota, examen, prueba, test, control... Como señalan Pérez, Pozo y Rodríguez (2003) en las aulas universitarias suelen predominar los enfoques de enseñanza unidireccional, basados en el dictado de apuntes y su fiel devolución posterior el día del examen. Freire (1990) denominaba a este modelo de enseñanza "Educación Bancaria". Varios autores coinciden en criticar la tradición universitaria según la cual entre dos y tres semanas de cada cuatrimestre se dedican exclusivamente a la realización de exámenes, cuya única finalidad es el control y la calificación (Gibbs, 2003; López-Pastor, 2008).

Recientemente Ibarra y Rodríguez-Gómez (2010) han analizado los estatutos y normativas específicas sobre evaluación de universidades, facultades y departamentos indicando que: (a)-la evaluación se utiliza principalmente para comprobar los conocimientos de los estudiantes, estando más orientada a los resultados que a los procesos; (b)-en los documentos oficiales de las universidades predomina el discurso tradicional, focalizado en la evaluación del rendimiento académico del estudiante y su calificación (exámenes, convocatorias, reclamaciones, etc.); (c)-la técnica evaluativa por excelencia sigue siendo el examen final; (d)-no aparecen referencias a la función formadora de la evaluación; (e)-no se regula la participación del alumnado en la evaluación, salvo para la revisión de exámenes o la reclamación de calificaciones.

En los últimos veinte años se han realizado algunos estudios sobre la percepción que tienen los alumnos del tipo de evaluación que se lleva a cabo en las aulas universitarias (Fernández-Pérez, 1989; Flórez, 1999; Gargallo, 2009; Rodríguez-Gómez et al., 1998; Tejedor, 1998; Trillo, 2005; Trillo y Porto, 1999). No obstante, como señalan Trillo y Porto (1999) son pocos los que se han realizado en profundidad para saber cómo se está desarrollando de manera específica la evaluación de los estudiantes universitarios. En ellos se constata que porcentajes muy elevados de alumnos perciben: (a)-fuertes contradicciones entre la evaluación y los procesos de enseñanza-aprendizaje (E-A); (b)por evaluar se entiende calificar a los estudiantes mediante un examen y decidir sobre su promoción; (c)-para examinar se emplea principalmente el examen escrito, sin poder consultar ningún tipo de material; (d)-en el proceso de evaluación sólo participa el profesor que imparte la materia, sin hablarla ni acordarla con los alumnos ni con los otros profesores con los que comparte la misma materia; (e)-la mayoría de los profesores no explicitan los criterios que siguen para evaluar; (f)-la evaluación solo se hace al final del proceso de E-A; (g)-las funciones principales de la evaluación son la selección de alumnos y la certificación de resultados.

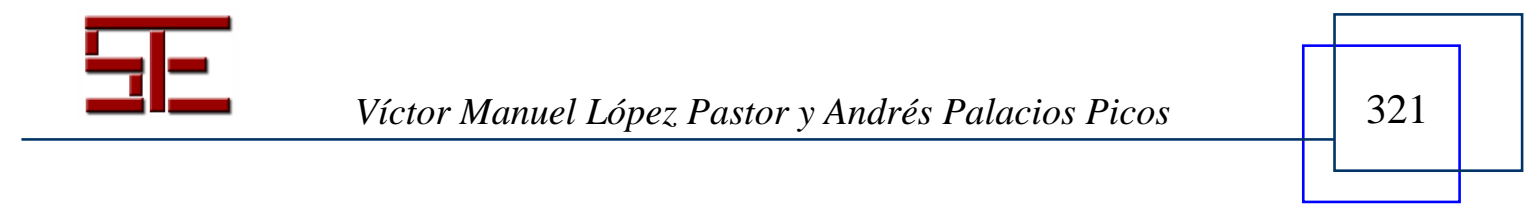




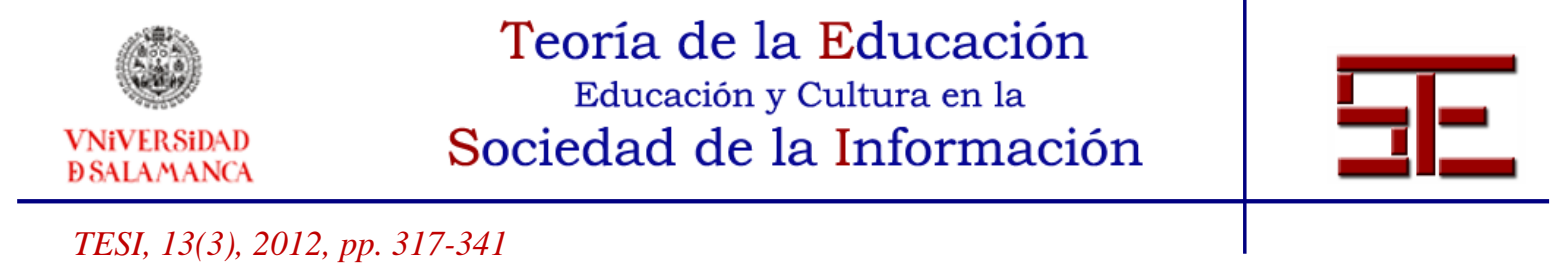

Rodríguez-Gómez et al. (1998) encuentran resultados similares en un estudio sobre la percepción que tiene el alumnado respecto a la evaluación de primer curso de diferentes carreras. Los datos muestran que: (a)-se evalúan principalmente conocimientos teóricos; (b)-se valora la adquisición memorística de conocimientos puntuales; (c)-no hay participación del alumnado en la evaluación; (d)-encuentran diferencias fuertes en el sistema de evaluación entre diferentes asignaturas; (e)-los instrumentos más utilizados fueron: trabajos escritos individuales, entrevista personal, pruebas tipo test, trabajos en grupo, prácticas y pruebas tipo ensayo; (f)-cuando se piden trabajos o bien no se tienen en cuenta en la calificación final o bien eran poco valorados, dado que casi todo el peso de la calificación final era para el examen; (g)-no existían criterios claros que respaldaran la calificación otorgada al trabajo; (h)-la evaluación no es de proceso, solo valora el producto final; (i)-no hay retroalimentación ni posibilidad de mejorar durante el proceso, no hay evaluación integrada, solo momentos puntuales de evaluación; (j)-se da un excesivo uso de exámenes tipo test.

En clara coincidencia con los datos anteriores, Gargallo (2009) concluye que en la universidad sigue siendo escaso el uso de la evaluación inicial o de diagnóstico para comprobar los conocimientos o competencias que el alumno posee antes de iniciar el proceso formativo, aunque hay un cierto avance en el uso de la evaluación formativa.

Fernández-Pérez (1989) encuentra que la mayoría del alumnado universitario reconoce que vive una evaluación exclusivamente puntual, basada en la realización de una única prueba (examen), normalmente de tipo memorístico y que suelen desconocerse los criterios que utilizan los profesores para calificar dicha prueba. El estudio de Gros y Romañá (1995) refuerza esta tesis, dado que encuentra evidencias de que la mayoría del profesorado usa el examen como única forma de evaluación, excepto en el caso de asignaturas con pocos estudiantes. Tejedor (1998), tras realizar un estudio con alumnos de último curso, concluye que el alumnado considera que en las aulas universitarias se evalúa básicamente para calificar y no para orientar el aprendizaje. En un estudio posterior, Trillo (2005) constata que el alumnado expresa graves críticas a la forma en que se lleva a cabo el proceso de evaluación en la universidad y explica que dichos resultados no parecen ser demasiado diferentes de los encontrados en el contexto anglosajón. Por su parte, Flórez (1999) encuentra que la evaluación es la dimensión docente más controvertida y la peor valorada, tanto para el alumnado como para los egresados. Zabalza (2003), tras revisar la literatura sobre la situación de la evaluación en la universidad española, señala que la actividad evaluadora es una de las carencias más extendidas en las aulas universitarias.

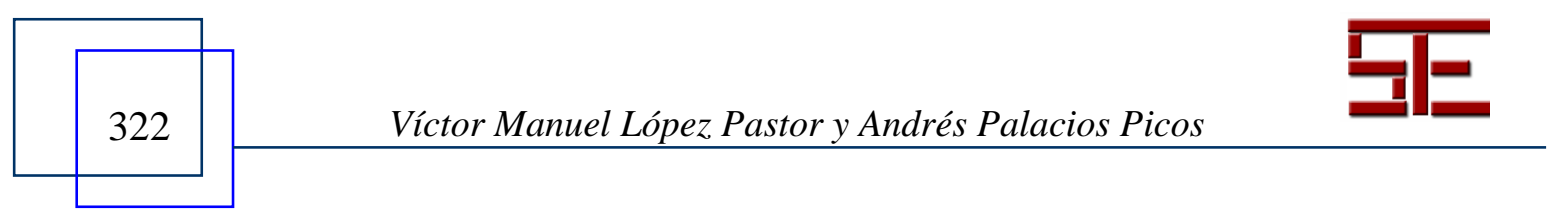




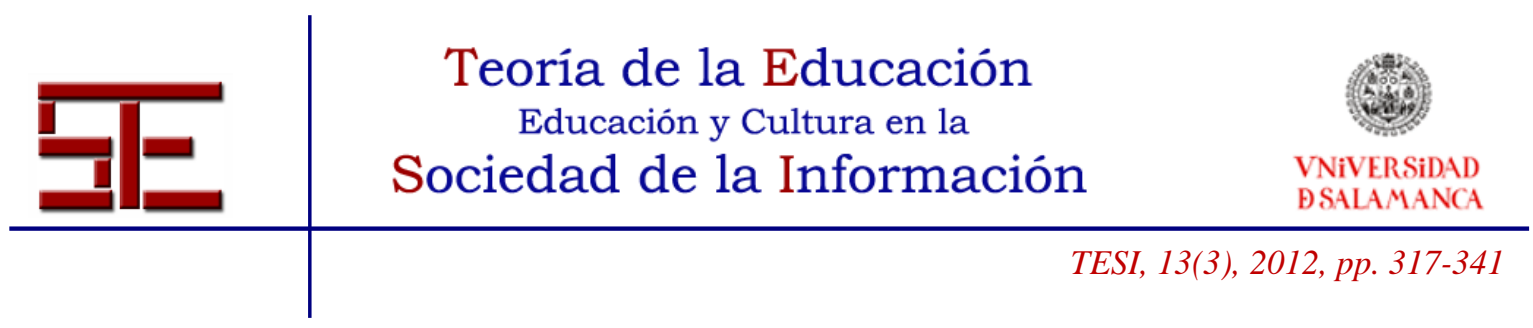

Centrándonos en los procesos de evaluación entre iguales (peer-assessment), McMahon (2010) encuentra que el alumnado rechaza estos procesos cuando tienen consecuencias en la calificación de sus compañeros. La mayor parte del alumnado muestra desinterés por este tipo de procesos y una parte del alumnado muestra también resistencias y negativas a realizarlos, porque consideran que ellos no deben calificar a sus compañeros, que se trata de una tarea que corresponde realizar al profesor y que les genera sentimientos negativos. En cambio, los procesos de evaluación entre iguales reciben una valoración positiva del alumnado universitario cuando se orientan exclusivamente a aportar feed-back positivo para mejorar el aprendizaje de sus compañeros, sin tener ningún efecto en la calificación de los mismos. De forma similar, López-Pastor (2009) encuentra que el alumnado universitario muestra resistencias en procesos de evaluación entre iguales cuando están orientados a la calificación, mientras que muestran una vivencia positiva si el proceso está orientado exclusiva, o principalmente, a la mejora del aprendizaje. Por su parte, Hamodi y López (2012), en un estudio que utiliza grupos de discusión, encuentran que el alumnado acepta más la evaluación colaborativa con el profesor que la evaluación entre iguales.

La universidad vive un periodo crucial en muchos aspectos. En las líneas anteriores hemos resumido investigaciones que muestran la trascendencia de realizar procesos de evaluación innovadores como mejora de la calidad del aprendizaje en la universidad. Pero también la dificultad de conocer lo que realmente sucede actualmente en las aulas universitarias.

El objetivo principal de este estudio es analizar la perspectiva que el alumnado tiene sobre los sistemas, instrumentos y técnicas de evaluación y calificación que se utilizan actualmente en la formación inicial del profesorado (FIP).

Este trabajo puede servir de línea base sobre los sistemas y técnicas de evaluación justo en el momento en el que se han generalizado los nuevos planes de estudio y se ha hecho realidad el proceso de convergencia hacia el EEES. Estudios posteriores podrán comparar lo alcanzado por estos nuevos planes a partir de las diferencias con lo ahora obtenido.

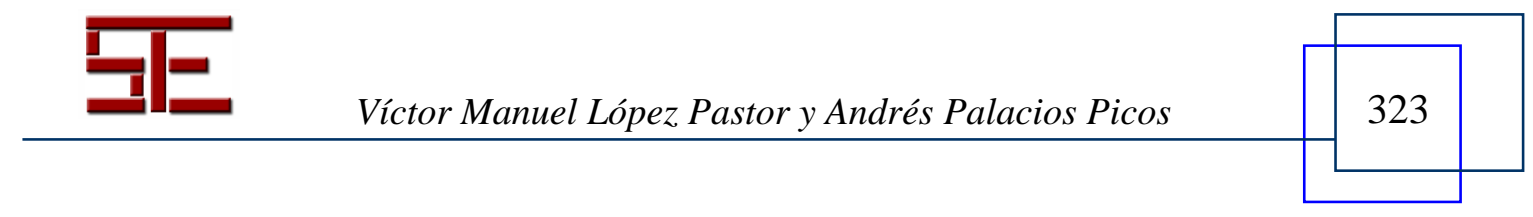




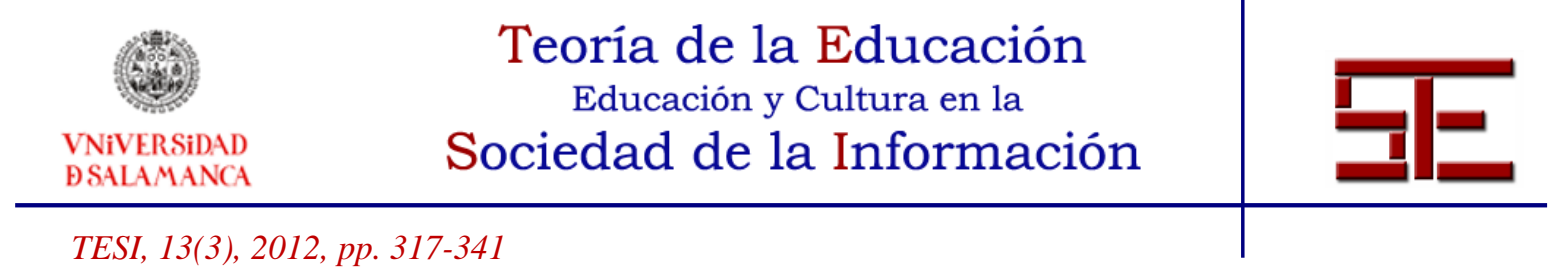

\section{2.- MATERIAL Y MÉTODO}

\section{1.- Participantes}

El estudio se ha llevado a cabo con una muestra de 635 alumnos de último curso de las titulaciones de Maestro (especialidades de Primaria, Musical, Educación Física, Lengua Extranjera e Infantil) y Licenciado en Ciencias de la Actividad Física y del Deporte (CCAFyD), de los campus universitarios de Ávila, León, Salamanca, Segovia, Valladolid y Zamora. La muestra presenta un predominio de alumnas (63\%), con una edad media de 22 años y con la distribución por titulaciones y campus que resumimos en el Cuadro 1.

Cuadro 1.- Distribución de la muestra por centros y especialidades-carreras

\begin{tabular}{|l|c|c|c|c|c|c|c|}
\hline & \multicolumn{7}{|c|}{ Especialidad } \\
\cline { 2 - 8 } & Primaria & Musical & Ed. Física & Ed. Infantil & $\begin{array}{c}\text { Lg. } \\
\text { Extran. }\end{array}$ & CAFyD \\
\hline E.U. Magisterio Segovia & 43 & 7 & 110 & 45 & 0 & 0 \\
\hline Fac. CCAFyD de León (CCAFyD) & 0 & 0 & 0 & 0 & 0 & 65 \\
\hline Fac. Educación Valladolid & 12 & 13 & 33 & 53 & 0 & 0 \\
\hline Fac. Educación de León & 36 & 9 & 27 & 42 & 25 & 0 \\
\hline E.U. Magisterio de Zamora & 0 & 0 & 40 & 17 & 0 & 0 \\
\hline E.U. Magisterio de Ávila & 0 & 7 & 0 & 0 & 0 & $\mathbf{6 5}$ \\
\hline Fac. Educación Salamanca & 26 & 0 & 0 & 25 & 0 & $\mathbf{1 1 5}$ \\
\hline TOTAL & $\mathbf{1 1 7}$ & $\mathbf{3 6}$ & $\mathbf{2 1 0}$ & $\mathbf{1 8 2}$ & $\mathbf{2 5}$ & $\mathbf{7}$ \\
\hline
\end{tabular}

\section{2.- Instrumentos}

La toma de datos se realizó mediante un cuestionario sobre diferentes aspectos relacionados con la evaluación y la calificación. Para su construcción se han tenido en cuenta instrumentos ya validados con temática parecida (Fernández-Pérez, 1989; Flórez, 1999; Trillo, 2005; Tejedor, 1998). La novedad de nuestro cuestionario estriba en que se analizan de manera conjunta tres aspectos del proceso evaluador como son los sistemas de evaluación, los sistemas de calificación y la participación del alumnado en dichos procesos.

En una primera fase, se dispuso de un conjunto de preguntas para cada uno de los tres apartados antes mencionados, con los que se elaboró el primer modelo de cuestionario.

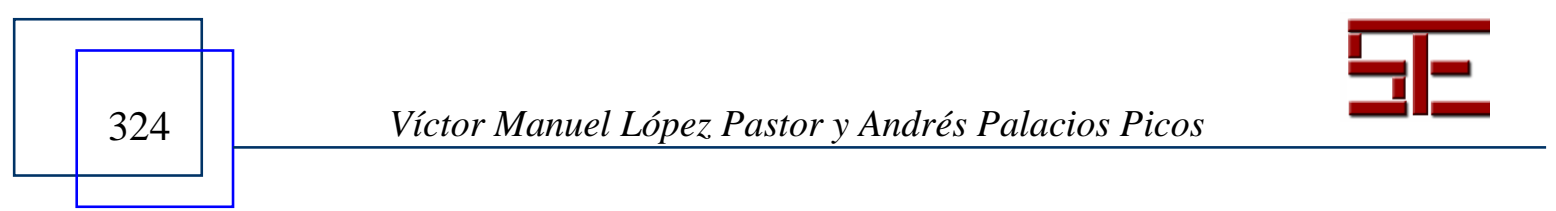




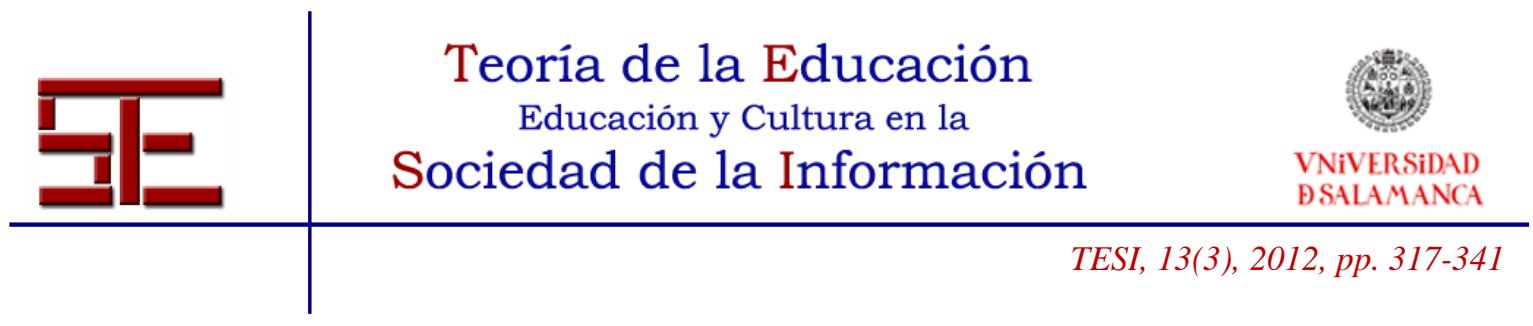

Este primer borrador fue evaluado por un total de 14 expertos profesionales de la FIP. De este primer cuestionario fueron seleccionadas las preguntas más pertinentes por su relevancia (los ítems deberían estar claramente relacionados con el objeto de estudio) y claridad (fácilmente comprensibles, con afirmaciones simples) con las que se realizaron los primeros pre-test con una pequeña muestra de alumnado, para asegurar su comprensión.

La versión final del cuestionario consta de 27 preguntas en cada una de las cuales el alumno responde según el grado de acuerdo con el enunciado en una escala tipo Likert de cinco puntos (valores de 0 a 4). A nivel de contenido, el cuestionario está dividido en los tres apartados antes mencionados: Características de la Evaluación (10 preguntas), Sistemas de Calificación y tipo de examen (13 preguntas) y Participación del Alumnado en el proceso de evaluación y calificación (4 preguntas). Pueden consultarse las 27 preguntas en los Cuadros 2, 3, 4, 5 y 6. Con el total de ítems de la escala se obtuvo un Alfa de Cronbach de .80, que puede ser considerado un valor alto para este tipo de instrumentos, lo que nos asegura que nuestras medidas y sus resultados sean fiables.

Por lo que respecta a la validez de contenido, se procedió a realizar un Análisis Factorial Confirmatorio (AFC). Para su cálculo, se utilizaron las matrices de covariaciones y el algoritmo de cálculo de los mínimos cuadrados no ponderados, pues con dicha estrategia de cálculo se realizan estimaciones consistentes sin necesidad de asumir multinormalidad de las variables (Ruiz, 2000). Concretamente, se ha contrastado la hipótesis de la existencia de los factores correspondientes a las 3 vertientes del cuestionario: valoración de la evaluación formativa, de los sistemas de evaluación y participación de los alumnos en la evaluación (Tabla I).

Tabla I. Evaluación del cuestionario mediante AFC

\begin{tabular}{cccccccc}
\hline & S-B & Nhi-cuadrado) \\
\hline $\begin{array}{c}\text { Modelo })(\mathrm{p}) \\
\text { factorial }\end{array}$ & $\begin{array}{c}161.19(53) \\
(\mathrm{p}=.00)\end{array}$ & .110 & .89 & .91 & .93 & .81 & 156.00 \\
\hline
\end{tabular}

Como podemos comprobar, el modelo alcanza un ajuste moderado. Como valor a resaltar la matriz LAMBDA-X presenta todos los valores significativos con $p<.05$, lo que proporciona evidencias añadidas de la validez de las preguntas de la escala.

Como complemento al cuestionario, se realizaron preguntas relativas a la edad, sexo, titulación en que realiza sus estudios, especialidad y titulación de acceso a los estudios.

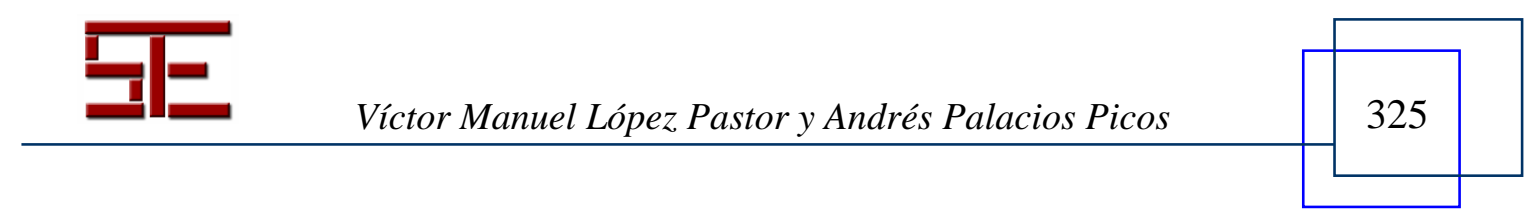




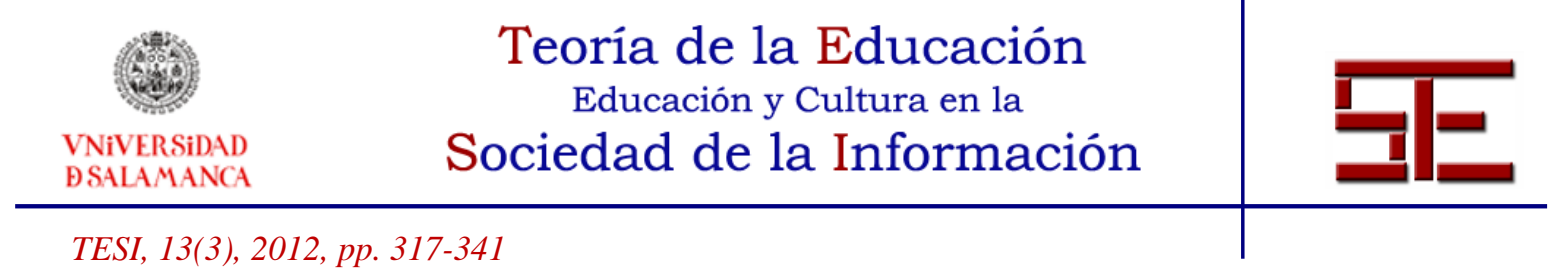

\section{3.- Procedimiento}

La administración de los cuestionarios se realizó por parte del equipo de investigación durante las últimas semanas del curso académico 2008/2009. Los cuestionarios tenían un carácter anónimo y fueron autocumplimentados por los sujetos de la muestra.

\section{4.- Análisis estadístico}

Los datos obtenidos fueron analizados mediante los paquetes estadísticos SPSS 15.0 y LISREL 8.7, este último para el Análisis Factorial Confirmatorio. Puesto que el objetivo fundamental del estudio es indagar sobre la percepción del alumnado, se ha trabajado fundamentalmente con las frecuencias relativas de cada una de las posibles respuestas. No obstante, se han realizado ANOVAS en determinadas preguntas para establecer el grado de significación estadística de las diferencias entre el alumnado proveniente de Magisterio el alumnado de la licenciatura en Ciencias de la Actividad Física y del Deporte.

\section{3.- RESULTADOS}

\section{1.- Características de la evaluación}

Un primer dato a comentar es la ausencia de una negociación curricular al comienzo de curso sobre el sistema de evaluación; concretamente, para la mitad de los alumnos encuestados no se ha producido discusión alguna; solo para 2 de cada 10 alumnos se han discutido los criterios de evaluación de manera habitual en su formación (Cuadro 2).

\begin{tabular}{|c|c|c|c|c|c|c|}
\hline \multirow{2}{*}{$\begin{array}{l}\text { PREGUNTAS } \\
\text { En cuántas asignaturas... }\end{array}$} & \multicolumn{4}{|c|}{ Porcentajes de respuestas } & \multirow[b]{2}{*}{$\begin{array}{c}\text { Muchas } \\
\text { (4) }\end{array}$} & \multirow[b]{2}{*}{$\begin{array}{c}\text { Media } \\
\text { aritmética }\end{array}$} \\
\hline & $\begin{array}{l}\text { Ninguna } \\
(0)\end{array}$ & $\begin{array}{l}\text { Pocas } \\
(1)\end{array}$ & $\begin{array}{c}\text { Algunas } \\
(2)\end{array}$ & $\begin{array}{c}\text { Bastantes } \\
(3)\end{array}$ & & \\
\hline Se discutió el sistema de evaluación al comienzo del curso & 17.9 & 33.1 & 29.4 & 14.6 & 5.0 & 1.74 \\
\hline Se realizaban procesos de evaluación continua & 5.5 & 25.4 & 38.6 & 26.2 & 4.3 & 1.99 \\
\hline Se utilizaban procesos de evaluación formativa & 17.8 & 40.3 & 29.3 & 11.7 & 1.0 & 1.38 \\
\hline $\begin{array}{l}\text { Las observaciones realizadas sobre los trabajos demandados } \\
\text { podían derivar en la repetición de los mismos para mejorarlos }\end{array}$ & 21.6 & 41.9 & 25.8 & 9.2 & 1.5 & 1.27 \\
\hline
\end{tabular}

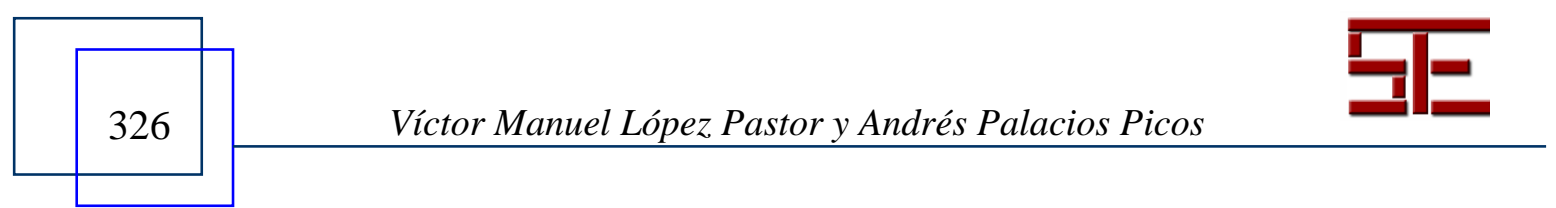




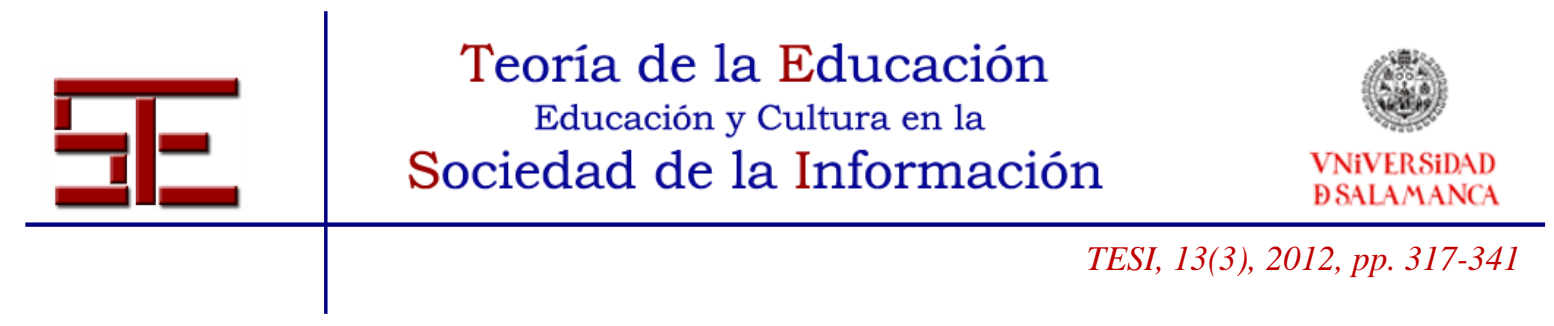

\begin{tabular}{|c|c|c|c|c|c|c|}
\hline Se utilizaban portafolios o carpeta individual & 20.7 & 33.2 & 27.9 & 14.1 & 4.2 & 1.48 \\
\hline Se utilizaban portafolios grupales & 27.7 & 35.4 & 24.8 & 9.1 & 2.9 & 1.24 \\
\hline $\begin{array}{l}\text { Se explicaban previamente los criterios de calidad de los } \\
\text { diferentes trabajos o exámenes }\end{array}$ & 7.2 & 29.9 & 37.1 & 21.9 & 3.9 & 1.85 \\
\hline $\begin{array}{l}\text { Existían similitudes entre los sistemas de evaluación de las } \\
\text { diferentes asignaturas }\end{array}$ & 4.6 & 16.3 & 39.9 & 32.5 & 6.6 & 2.20 \\
\hline $\begin{array}{l}\text { Se comentaban los resultados y las dudas de los exámenes y } \\
\text { actividades de evaluación }\end{array}$ & 16.0 & 30.1 & 37.2 & 13.8 & 2.9 & 1.57 \\
\hline En cuántas asignaturas se evaluó el programa & 35.2 & 31.9 & 20.8 & 9.5 & 2.6 & 1.12 \\
\hline
\end{tabular}

Además, en opinión de una parte importante del alumnado, existirían pocas asignaturas en las que se hayan hecho explícitos al comienzo del curso los criterios de calidad de la participación de los estudiantes (exámenes, trabajos, prácticas, etc). Solo el 25\% de los encuestados manifiestan haber tenido de manera habitual esta posibilidad; para el $37 \%$ del alumnado no se explicitaron como norma estos criterios.

La ausencia tanto de un proceso de negociación de los sistemas de evaluación como del conocimiento de los criterios de calidad podría tener relación con un dato de interés: para un $62 \%$ del alumnado no se evaluaron los programas de las asignaturas. De hecho, las correlaciones entre las respuestas a las tres preguntas que acabamos de comentar son, en todos los casos, significativas $(\mathrm{r}=.63$, sig $=.00$, entre discusión sistema de evaluación y explicitación de criterios de calidad; $\mathrm{r}=.31$, sig=.00 entre discusión sistema de evaluación y la evaluación del programa; $\mathrm{r}=.48$, sig=.00; $\mathrm{r}=.39$, sig=.00, entre explicitación de criterios de calidad y la evaluación del programa); es decir, en una parte importante de asignaturas ni se evaluó el programa, ni se hicieron explícitos los criterios de calidad, ni se consensuaron los criterios de evaluación.

La presencia de portafolios o carpetas, tanto individuales como grupales, como sistemas de evaluación es testimonial: dos de cada diez alumnos afirman haber utilizado las individuales y uno de cada diez las grupales de manera más o menos habitual. Ambas estrategias tienden a realizarse de manera simultánea y correlacionan de manera significativa con el uso de procesos de evaluación formativa $(\mathrm{r}=.62$, sig=.00, entre portafolio individual y grupal; $\mathrm{r}=.38$, sig=.00 entre portafolio individual y evaluación formativa; $r=.43$, sig=.00, entre portafolio grupal y evaluación formativa).

La realización y entrega de documentos durante el desarrollo de la asignatura es una práctica que, en opinión del 30\% de los alumnos, está presente en una parte importante

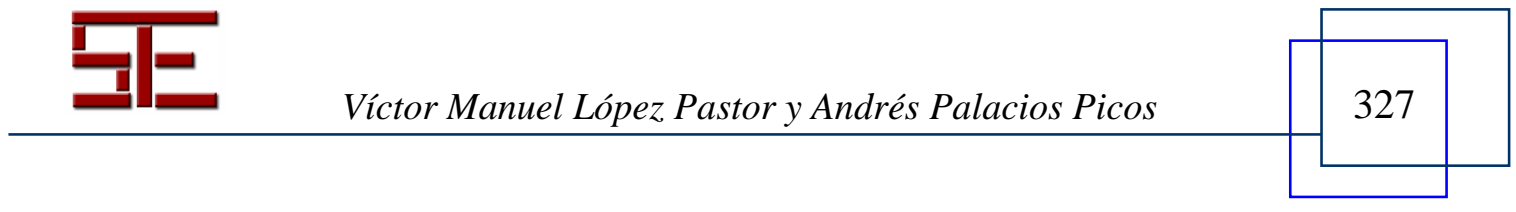




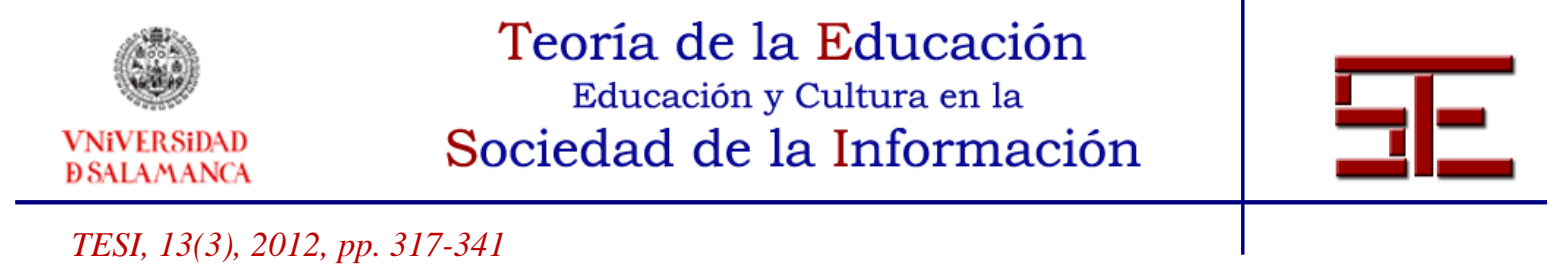

de asignaturas; aunque para otro $30 \%$ esta práctica ha estado ausente de su experiencia como alumno. Además, en estos casos en los que se elaboraban actividades a lo largo del curso, para un $63 \%$ no se realizaron procesos de feed-back. Solo para un $10 \%$ las observaciones realizadas por el profesor podrían derivar de manera habitual en la repetición de las actividades y los trabajos con el propósito de mejorarlos. Por otra parte, solo en la opinión de un $16 \%$ del alumnado se comentaban los resultados y las dudas de los exámenes y actividades.

Por todo lo dicho, no es extraño que solo uno de cada diez alumnos considere que, a lo largo de su carrera, se han utilizado procesos de evaluación formativa de manera generalizada. Resultados parecidos se dan en las opiniones sobre el uso de procesos de evaluación continua. No en vano, la correlación entre ambas variables es alta y significativa $(r=.44$, sig=.00). No obstante, existe un cierto acuerdo en que hay diferencias importantes entre asignaturas; solo un $20 \%$ no encontraría diferencia alguna en relación con los sistemas de evaluación utilizados entre las asignaturas cursadas.

Como venimos comentando, los valores medidos en cada una de las preguntas son bajos. Pero se hacen aún más bajos en los futuros docentes de secundaria (licenciados), si se analizan de manera separada sus respuestas y las de los futuros maestros (diplomados) (Figura 1).

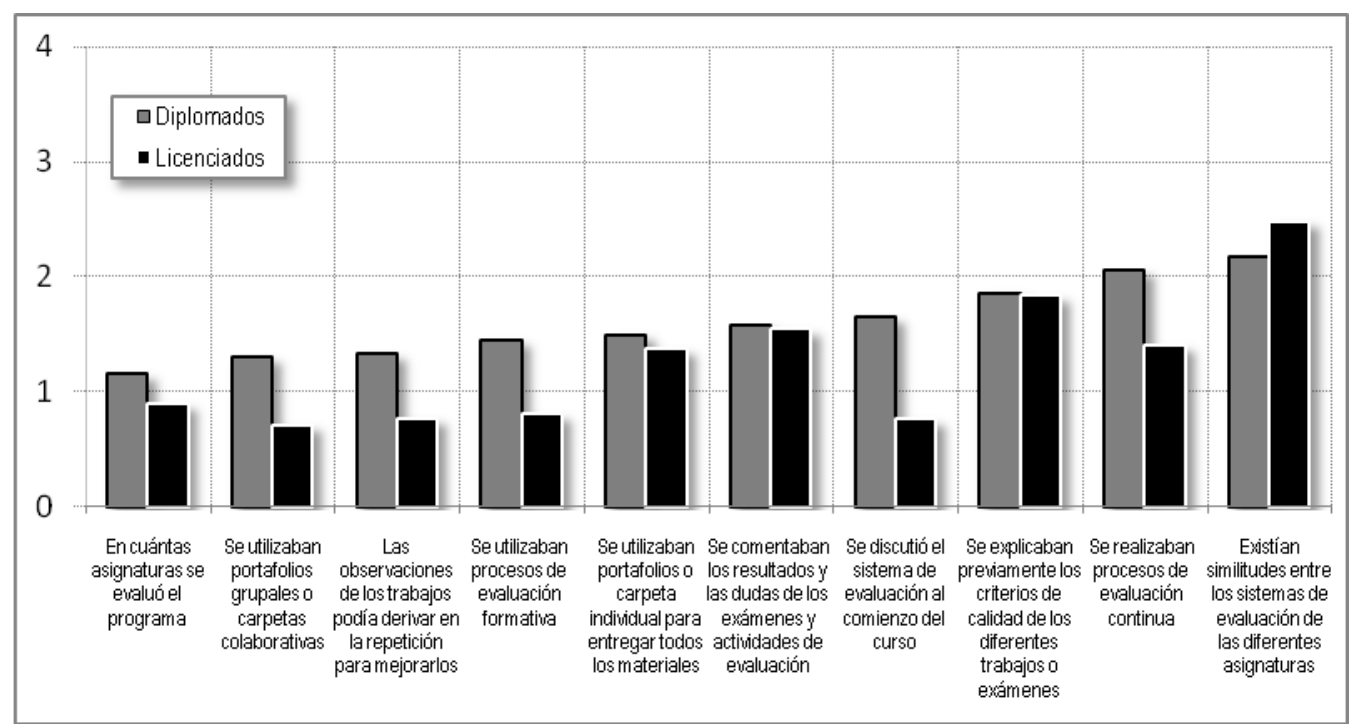

Figura 1.- Valor medio de las características de la evaluación (por tipo de carrera)

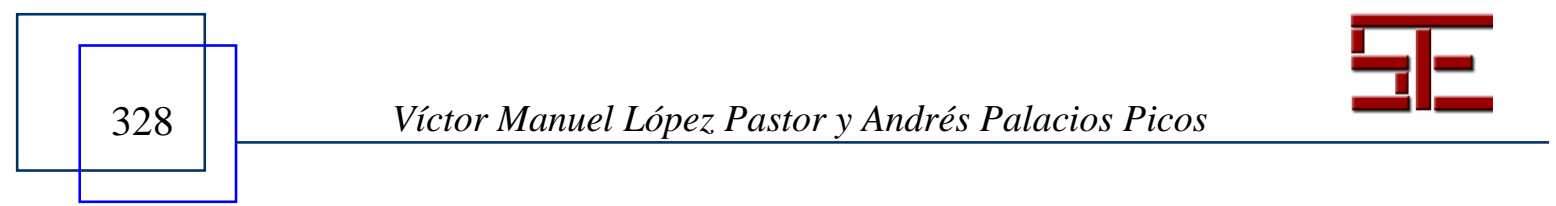




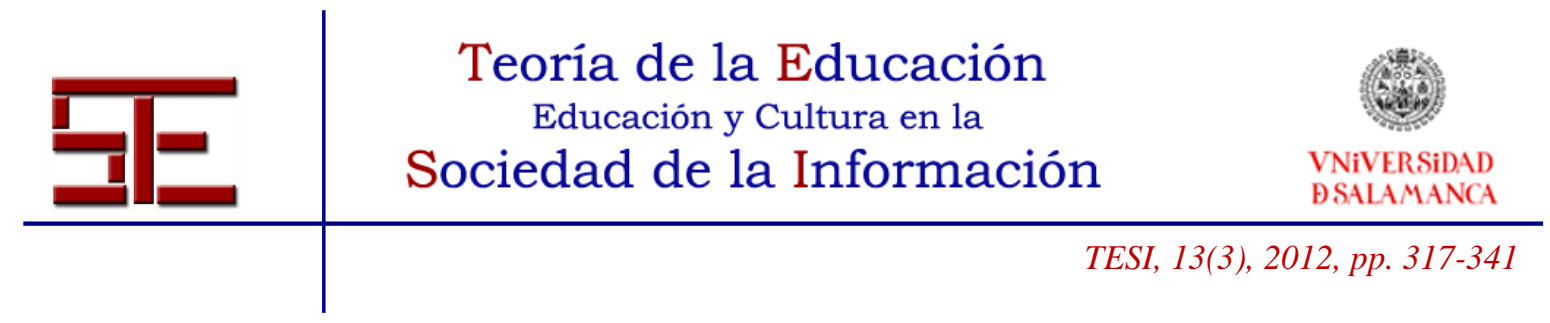

\subsection{Tipo de examen}

En opinión de los alumnos encuestados, el tipo de examen más frecuente es el desarrollo escrito de preguntas largas sobre un tema concreto (exámenes escritos de desarrollo). El valor medio de 3 puntos en una escala de cero -uso nulo-, a 4 -muy frecuentemente- así lo avala (Figura 2); este valor supone que el $71 \%$ de los entrevistados considera este examen escrito como el más utilizado de los posibles sistemas de evaluación y calificación.

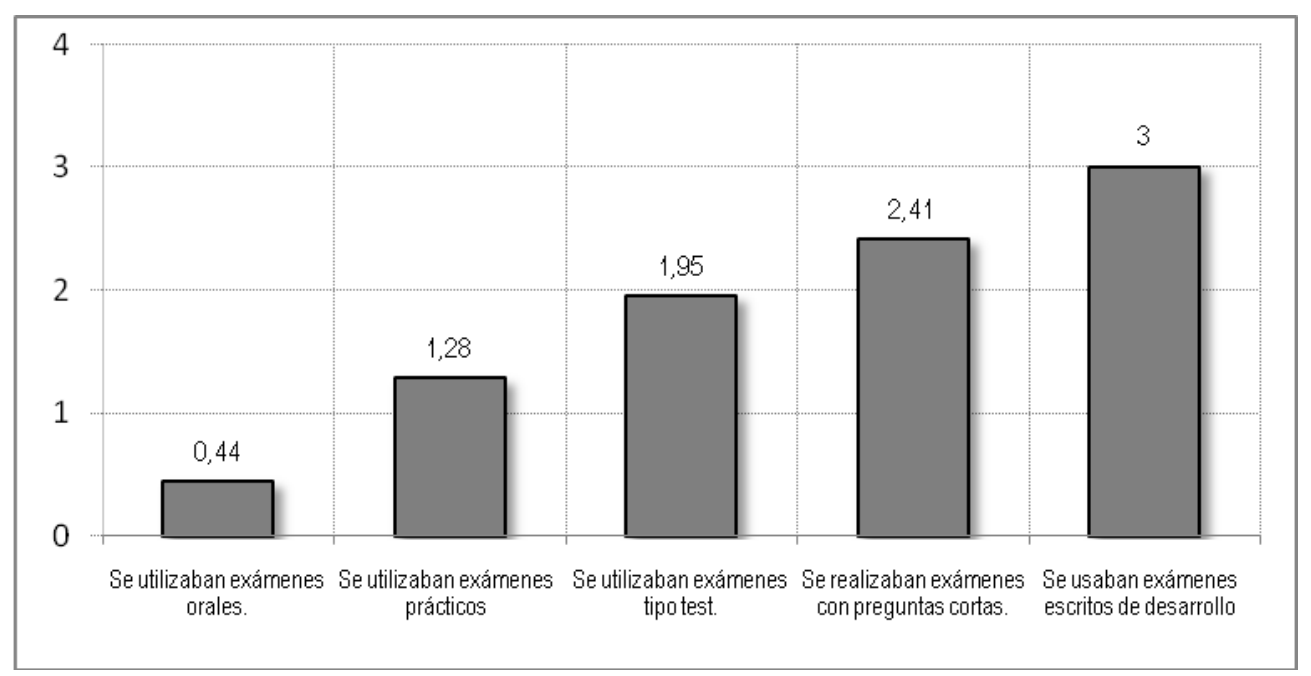

Figura 2.- Percepción de uso de diferentes tipos de exámenes.

Como segundo tipo más frecuente de examen, encontramos los basados en preguntas cortas, seguido de los exámenes tipo test, que aparecen señalados en el $25 \%$ de los alumnos encuestados. Entre los menos frecuentes, con un uso prácticamente testimonial, encontramos los exámenes orales: solo 2 de cada 100 alumnos los han realizado de manera habitual a lo largo de su formación; el $80 \%$ no los ha tenido nunca o casi nunca. Dato parecido al obtenido en relación al uso de exámenes prácticos mediante resolución de problemas, estudio de casos o planteamiento de actividades; ahora, solo 1 de cada 10 dice haber tenido dicho tipo de exámenes de manera habitual en sus años de formación; el $62 \%$ no los ha realizado nunca o casi nunca (Cuadro 3).

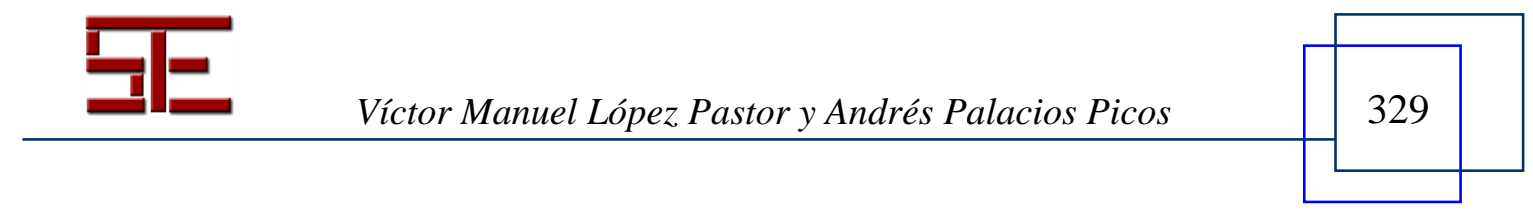




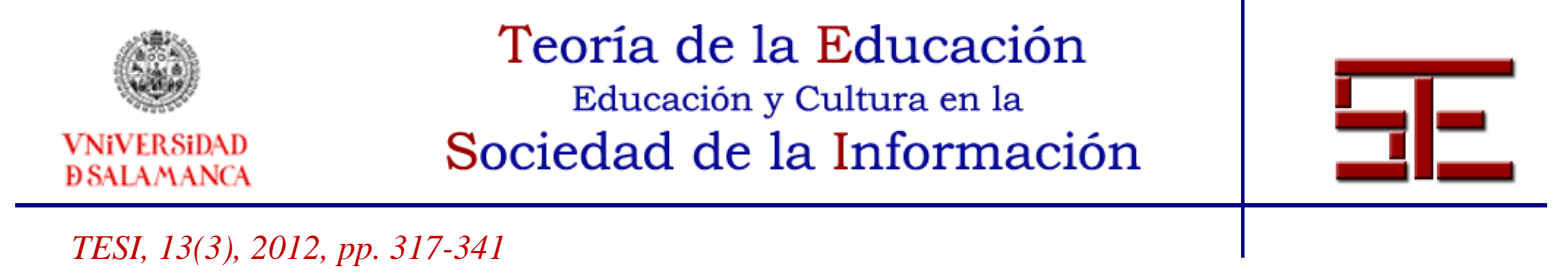

Cuadro 3.- Percepción de uso de diferentes tipos de exámenes

\begin{tabular}{|c|c|c|c|c|c|c|}
\hline \multirow{2}{*}{$\begin{array}{c}\text { PREGUNTAS } \\
\text { En cuántas asignaturas... }\end{array}$} & \multicolumn{4}{|c|}{ porcentajes de respuestas } & \multirow[b]{2}{*}{ Muchas } & \multirow{2}{*}{$\begin{array}{c}\text { Media } \\
\text { aritmética }\end{array}$} \\
\hline & Ninguna & Pocas & Algunas & Bastantes & & \\
\hline Se utilizaban exámenes orales & 69.7 & 18.9 & 8.8 & 2.4 & 0.2 & 0.44 \\
\hline Se usaban exámenes escritos de desarrollo & 1.4 & 7.4 & 20.4 & 31.1 & 39.7 & 3 \\
\hline Se realizaban exámenes con preguntas cortas & 1.9 & 13.8 & 38.7 & 32.5 & 13.1 & 2.41 \\
\hline Se utilizaban exámenes tipo test & 4.5 & 30.0 & 40.1 & 16.5 & 8.9 & 1.95 \\
\hline Se utilizaban exámenes prácticos & 22.3 & 38.8 & 28.7 & 9.0 & 1.1 & 1.28 \\
\hline
\end{tabular}

Como sucediera en el apartado anterior, encontramos diferencias al respecto del tipo de examen al comparar las opiniones de los futuros maestros y los futuros profesores de secundaria $(\mathrm{F}(1,624)=138.83$, sig=.00); en este último colectivo hay un mayor porcentaje de uso de los exámenes tipo test, de exámenes prácticos y de exámenes de preguntas cortas (Figura 3).

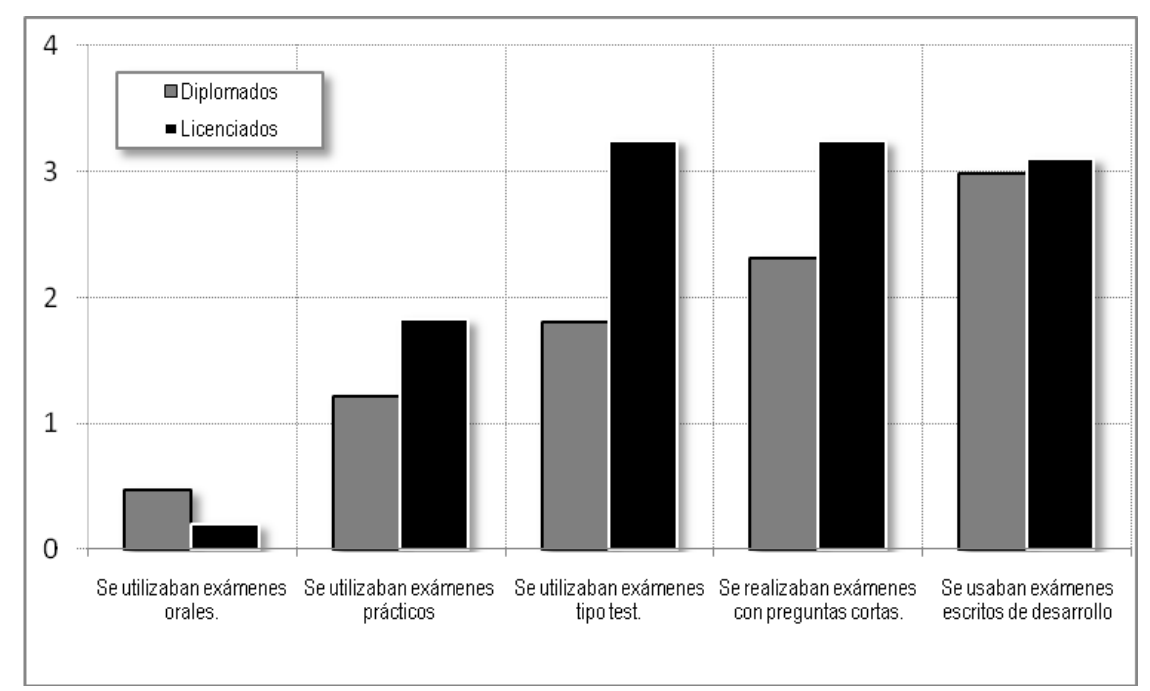

Figura 3.- Percepción de uso de diferentes tipos de exámenes (por tipo de carrera)

\section{2.- Sistemas de calificación}

Las opiniones del alumnado en este apartado son casi unánimes: a pesar de la posibilidad de realizar trabajos teóricos y prácticos, el examen final tiene un peso definitivo en la nota (Cuadro 4).

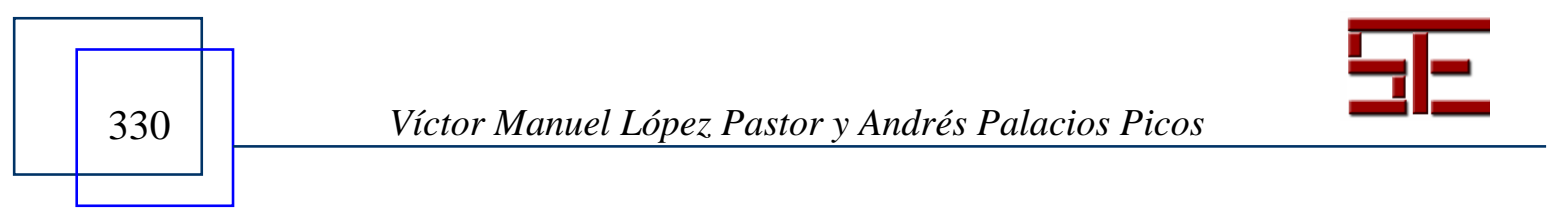




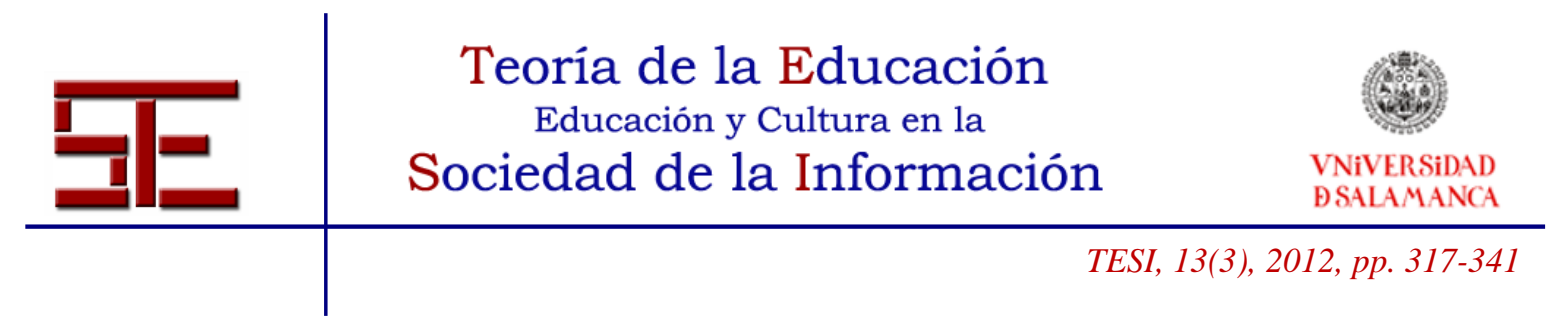

Cuadro 4.- Percepción de uso de diferentes sistemas de calificación (en porcentajes)

\begin{tabular}{|c|c|c|c|c|c|c|}
\hline & \multicolumn{4}{|c|}{ porcentajes de respuestas } & \multirow[b]{2}{*}{ Muchas } & \multirow{2}{*}{$\begin{array}{c}\text { Media } \\
\text { aritmética }\end{array}$} \\
\hline En cuántas asignaturas ... & Ninguna & Pocas & Algunas & Bastantes & & \\
\hline La calificación provenía únicamente del examen final & 3.2 & 11.7 & 29.6 & 34.7 & 20.8 & 2.58 \\
\hline La nota final provenía del examen final y algún trabajo & 1.3 & 5.3 & 31.1 & 42.2 & 20.1 & 2.74 \\
\hline $\begin{array}{l}\text { La calificación provenía del examen final y otros } \\
\text { instrumentos (cuadernos, trabajos en grupo, etc.) }\end{array}$ & 3.2 & 20.2 & 37.7 & 29.8 & 9.1 & 2.21 \\
\hline $\begin{array}{l}\text { La calificación final era la suma de las calificaciones de } \\
\text { los exámenes parciales realizados }\end{array}$ & 12.8 & 30.1 & 38.0 & 14.7 & 4.3 & 1.68 \\
\hline La calificación se obtenía sin exámenes & 39.2 & 41.1 & 16.7 & 2.8 & .3 & 0.84 \\
\hline $\begin{array}{l}\text { El sistema y los criterios de calificación se explicaban al } \\
\text { principio de la asignatura y eran conocidos }\end{array}$ & 3.4 & 18.4 & 36.3 & 29.0 & 13.0 & 2.30 \\
\hline La nota que ponía el profesor es justificada & 8.4 & 20.1 & 42.8 & 25.6 & 3.2 & 1.95 \\
\hline $\begin{array}{l}\text { A pesar de realizar diferentes trabajos teórico-prácticos, } \\
\text { el examen tenía un peso definitivo en la nota final }\end{array}$ & 1.4 & 5.4 & 13.8 & 34.6 & 44.7 & 3.16 \\
\hline
\end{tabular}

En consonancia con el dato anterior, algo más del $85 \%$ del alumnado considera que la calificación final provenía exclusivamente del examen final; porcentaje que se eleva a algo más del $93 \%$ cuando a esta nota final se le suma la de algún trabajo individual, cuaderno, práctica o trabajo en grupo; este sistema es el más extendido en opinión del alumnado.

La prevalencia del examen final como fuente de calificación es mayor entre los licenciados (valor medio de 3.20) que en los diplomados (valor medio de 2.51) ( $\mathrm{F}(1$, $623)=26.51$, sig=.00). En la mayoría de casos de asignaturas anuales, esa nota final se mantiene como sistema de calificación sin la presencia de exámenes parciales. En relación a la justificación de la nota emitida por el profesor, el 29\% manifiesta que era práctica habitual que la nota fuera explicada y razonada. No obstante, otro $29 \%$ manifiesta que en ningún caso se realizó justificación alguna. Sin embargo, el porcentaje de los que consideran que han hecho explícitos al comienzo de curso los sistemas de calificación sube al $42 \%$; el $22 \%$ opina que nunca o casi nunca se hicieron explícitos dichos criterios en las asignaturas cursadas. Donde hay una unanimidad casi absoluta es en la opinión de haber cursado muy pocas asignaturas en las que la calificación se obtuviera sin exámenes; solo 3 de cada 100 alumnos han vivenciado este modelo de calificación sin exámenes; todos ellos estudiantes de magisterio.

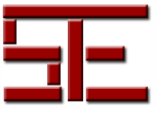

Víctor Manuel López Pastor y Andrés Palacios Picos 


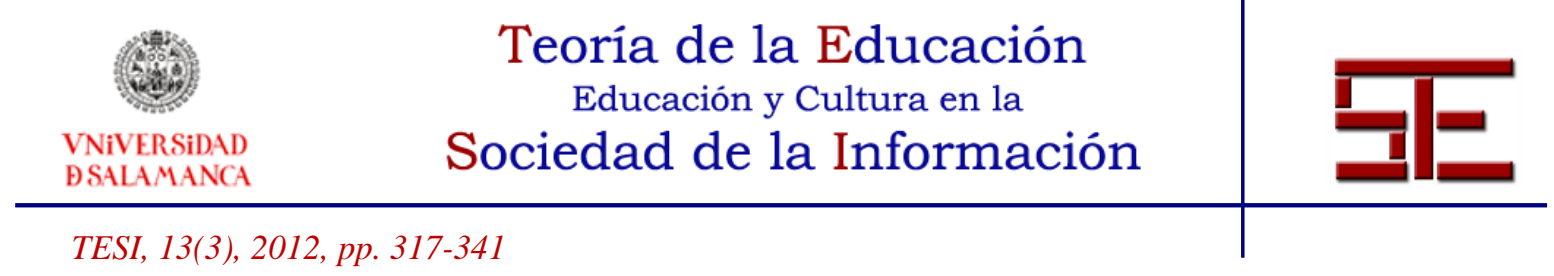

Como hemos señalado anteriormente, las diferencias entre licenciados y diplomados son significativas en la mayor parte de las preguntas de este apartado de sistemas de calificación (Cuadro 5).

Cuadro 5.- Diferencias de medias por tipo de carrera en las preguntas sobre sistemas de calificación

\begin{tabular}{|l|c|c|c|c|c|}
\multicolumn{1}{c}{$\begin{array}{c}\text { PREGUNTAS } \\
\text { En cuántas asignaturas... }\end{array}$} & \multicolumn{2}{c|}{ Valores medios por tipo de carrera } & \multicolumn{2}{c|}{ ANOVA } \\
\cline { 2 - 7 } & Diplomados & Licenciados & Media total & F & Sig. \\
\hline La calificación provenía únicamente del examen final & 2.51 & 3.20 & 2.58 & 26.51 & .00 \\
\hline La nota final provenía del examen final y algún trabajo & 2.70 & 3.15 & 2.74 & 16.03 & .00 \\
\hline $\begin{array}{l}\text { La calificación provenía del examen final y otros } \\
\text { instrumentos (cuadernos, trabajos en grupo, etc.) }\end{array}$ & 2.22 & 2.15 & 2.21 & 0.28 & .59 \\
\hline $\begin{array}{l}\text { La calificación final era la suma de las calificaciones de } \\
\text { los exámenes parciales realizados }\end{array}$ & 1.63 & 2.09 & 1.69 & 12.39 & .00 \\
\hline La calificación se obtenía sin exámenes & 0.87 & 0.55 & 0.84 & 8.94 & .00 \\
\hline $\begin{array}{l}\text { El sistema y los criterios de calificación se explicaban al } \\
\text { principio de la asignatura y eran conocidos }\end{array}$ & 2.32 & 2.08 & 2.30 & 3.41 & .06 \\
\hline La nota que ponía el profesor es justificada & 1.96 & 1.85 & 1.95 & 0.88 & .34 \\
\hline $\begin{array}{l}\text { A pesar de realizar diferentes trabajos teórico- } \\
\text { prácticos, el examen tenía un peso definitivo en la nota } \\
\text { final }\end{array}$ & 3.11 & 3.55 & 3.16 & 12.79 & .00 \\
\hline
\end{tabular}

\section{4.- Participación de los alumnos en los procesos de evaluación y calificación}

Los valores medios menores de todo el cuestionario se obtienen en este apartado dedicado a la participación estudiantil en los procesos de evaluación y calificación (Cuadro 6).

Cuadro 6.- Percepción de la participación estudiantil en los procesos de evaluación y calificación

\begin{tabular}{|c|c|c|c|c|c|c|}
\hline \multirow[b]{2}{*}{$\begin{array}{l}\text { PREGUNTAS } \\
\text { En cuántas asignaturas ... }\end{array}$} & \multicolumn{5}{|c|}{ porcentajes de respuestas } & \multirow[b]{2}{*}{$\begin{array}{c}\text { Media } \\
\text { aritmética }\end{array}$} \\
\hline & Ninguna & Pocas & Algunas & Bastantes & Muchas & \\
\hline $\begin{array}{l}\text { Los alumnos realizaban una valoración del } \\
\text { trabajo realizado por ellos mismos } \\
\text { (autoevaluación) }\end{array}$ & 29.3 & 40.7 & 26.6 & 2.9 & .5 & 1.04 \\
\hline $\begin{array}{l}\text { Los alumnos evaluaban los trabajos de sus } \\
\text { compañeros (coevaluación) }\end{array}$ & 39.3 & 39.4 & 19.1 & 1.9 & .3 & 0.85 \\
\hline $\begin{array}{l}\text { La calificación final era consensuada entre el } \\
\text { profesor y el alumno (calificación dialogada) }\end{array}$ & 55.1 & 33.7 & 9.8 & 1.3 & .2 & 0.58 \\
\hline $\begin{array}{l}\text { Eran los propios alumnos los que emitían una } \\
\text { calificación de manera justificada } \\
\text { (autocalificación) }\end{array}$ & 64.4 & 25.2 & 8.7 & 1.6 & .2 & 0.48 \\
\hline
\end{tabular}

332 Victor Manuel López Pastor y Andrés Palacios Picos




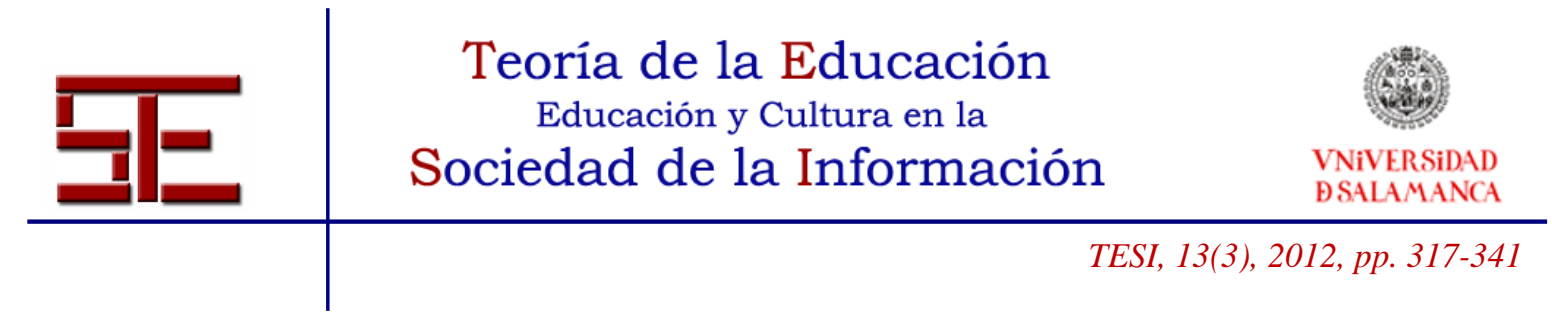

Volvemos a encontrar diferencias significativas $(\mathrm{F}(1,629)=54.71$, sig=.00) en la comparación del alumno de licenciaturas y diplomaturas (Figura 4).

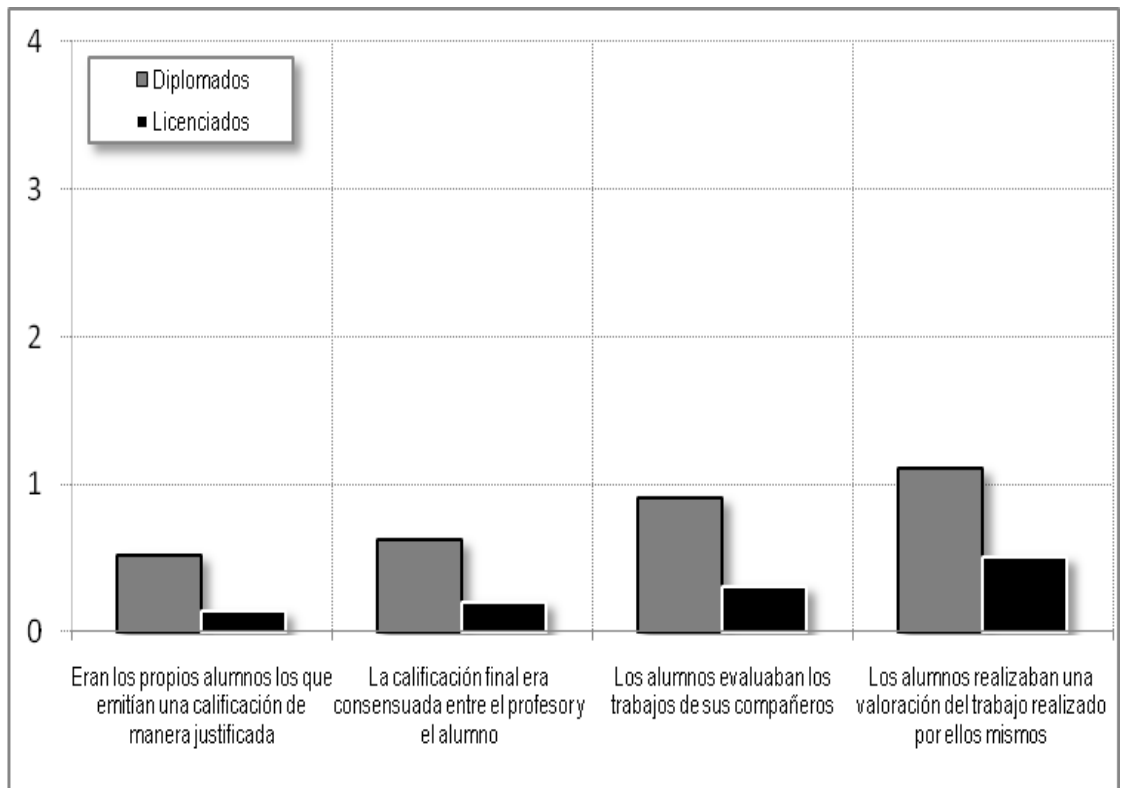

Figura 4.- Percepción de la participación estudiantil en los procesos de evaluación y calificación (por tipo de carrera)

No obstante estas diferencias, el dato más relevante a comentar son las bajas valoraciones de todos los encuestados, sin distinción de especialidad o carrera, al respecto de la participación del alumnado en los procesos de evaluación y calificación. Pongamos un ejemplo; solo en la opinión de un 3\% han tenido la oportunidad de realizar una autoevaluación de manera habitual a lo largo de su formación. Estos bajos valores se repiten al respecto de la posibilidad de evaluar los trabajos de los compañeros (coevaluación) o de realizar una calificación final consensuada con el profesor (calificación dialogada). La posibilidad de realizar un proceso de autocalificación solo ha estado presente de manera habitual en su formación para un $0.2 \%$; la cifra sube a un $1 \%$ de los que han realizado esta autocalificación de manera ocasional.

Las altas correlaciones que se obtienen en las cuatro preguntas relacionadas con la participación del alumnado (autoevaluación, coevaluación, autocalificación y calificación dialogada) hacen suponer que, cuando se dan, suelen ser en las mismas asignaturas, pero en muy pocas asignaturas (Cuadro 7).

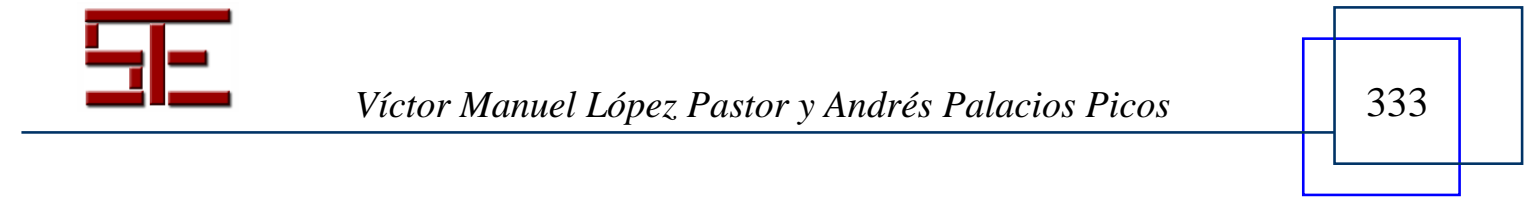




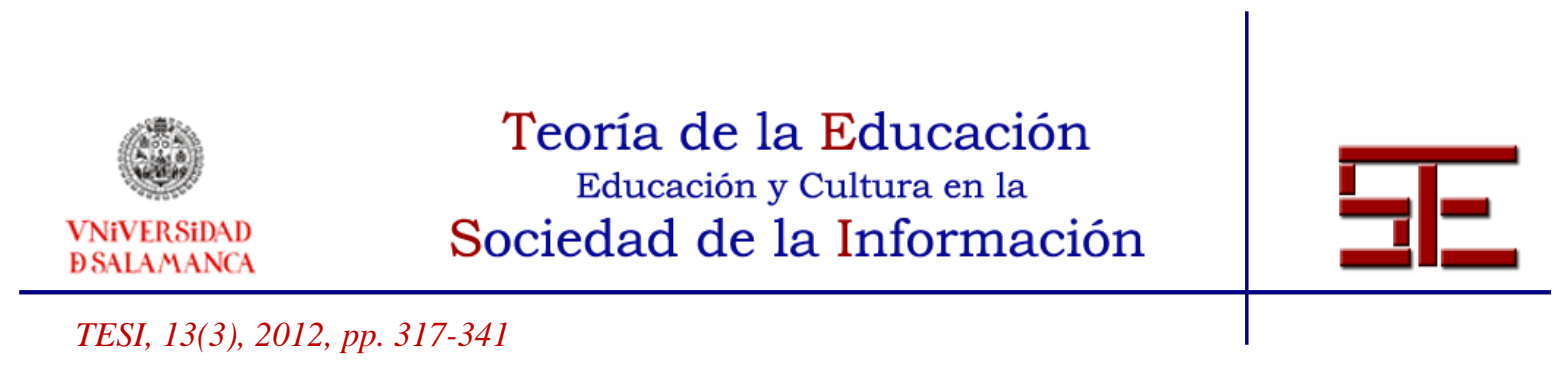

Cuadro 7.- Correlaciones entre las preguntas del apartado de participación estudiantil

\begin{tabular}{|c|c|c|c|c|}
\hline & autoevaluación & coevaluación & calificación & autocalificación \\
\hline autoevaluación & 1 & & & \\
\hline coevaluación & $.58^{* *}$ & 1 & & \\
\hline calificación dialogada & $.42^{* *}$ & $.46^{*}$ & 1 & \\
\hline autocalificación & $.31^{* *}$ & $.44^{* *}$ & $.61^{* *}$ & 1 \\
\hline
\end{tabular}

En este mismo sentido, cuando analizamos conjuntamente estas preguntas de participación del alumnado en los procesos de evaluación con otras relacionadas con sistemas innovadores de evaluación obtenemos altas correlaciones (preguntas sobre obtener la calificación sin exámenes, y utilizar procesos de evaluación formativa y de evaluación continua). Podemos suponer, como antes lo hicimos, que existen asignaturas con estilos de evaluación orientados al aprendizaje, en las que se utilizan estrategias variadas que hacen que aumenten los porcentajes de uso, dando la falsa imagen de una mayor utilización de lo que realmente se usa.

\section{DISCUSIÓN}

De manera mayoritaria, los alumnos universitarios siguen sin percibir cambios en sus sistemas de evaluación y calificación, al menos en línea con una evaluación formativa: (a)-apenas se discuten los criterios de evaluación y de calificación al comienzo de curso; (b)-no se evalúan los programas de las asignaturas; (c)-en muy pocas ocasiones se realizan procesos de feed-back de las actividades de aula, no hay retroalimentación ni posibilidad de mejorar durante el proceso; (d)-no hay evaluación integrada, solo momentos puntuales de evaluación; e)-el examen final escrito de desarrollo sigue siendo el principal sistema de calificación y, en muchos casos, el único; (f)-son testimoniales los casos en los que se califica sin exámenes, así como la participación del alumnado en los procesos de evaluación y calificación. Estos resultados coinciden con los estudios realizados sobre la temática en las dos últimas décadas (Fernández-Pérez, 1989; Flórez, 1999; Nieto, 2000; Trillo, 2005; Trillo y Porto, 1999).

A pesar de esta tónica general, parece existir un pequeño porcentaje de asignaturas que muestran lo que hemos denominado una Evaluación Orientada al Aprendizaje (EOA), caracterizada por tener una finalidad predominantemente formativa y continua, utilizar los exámenes finales como fuente complementaria de calificación, con una información

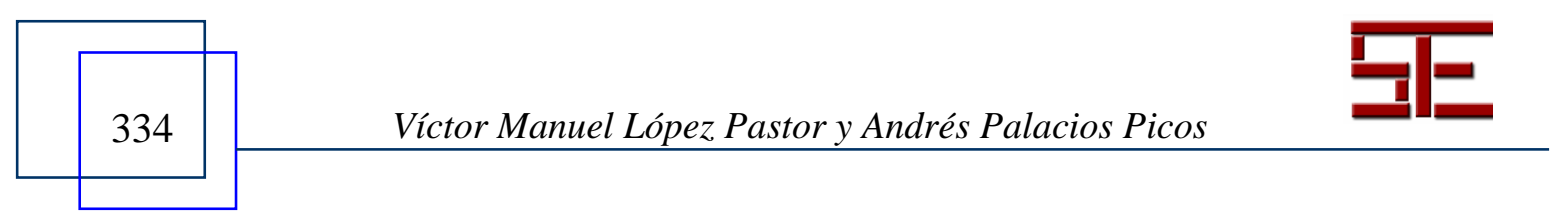




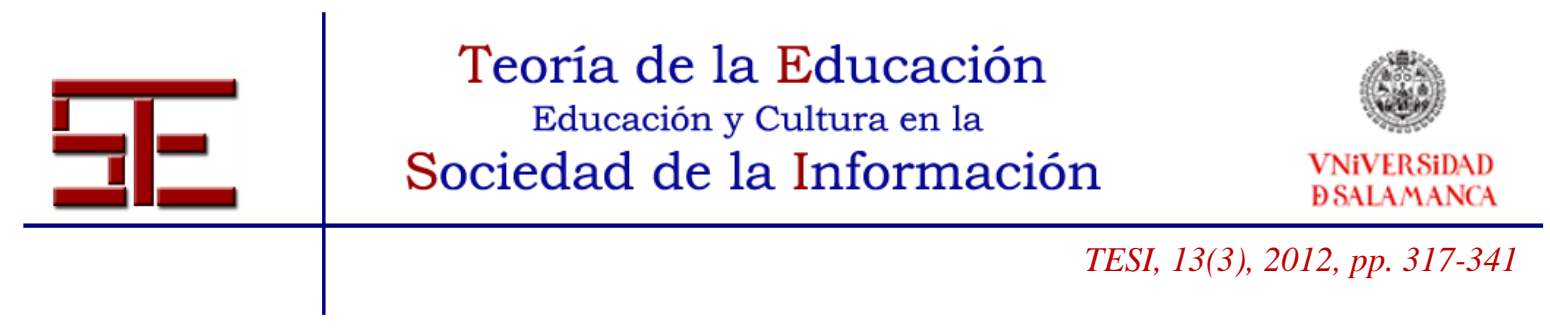

explícita de los criterios de calificación utilizados y con negociación de los mismos al principio de la asignatura; en algunos casos se promueve la participación del alumnado en los procesos evaluativos y suelen utilizarse portafolios o carpetas como herramienta recopilatoria del proceso de aprendizaje y evaluación. Características que coinciden con las indicadas por los principales especialistas (Boud y Falchikov, 2007; Carless, Joughin y Mok, 2006, Falchikov, 2005), así con algunos estudios y experiencias innovadoras en FIP (Fernández-Balboa, 2006; López-Pastor, 2009; Martínez, Martín y Capllonch, 2009; Santos, Martínez y López, 2009; Tabernero, De las Heras y González-Boto, 2009). Rodríguez-Gómez et al. (1998), en un estudio realizado con el alumnado de primer curso, resaltan la presencia de un número significativo de profesores que combinan la realización de actividades a lo largo del curso con un examen final, situación que supone un cierto cambio respecto a estudios de una década anterior (Nieto, 2000; Tejedor, 1998; Trillo y Porto, 1999), en los cuales el porcentaje de alumnado que consideraba que la calificación provenía exclusivamente del examen final solía situarse en torno al $90-95 \%$, frente al $83 \%$ de nuestros datos. También parece incrementarse la utilización de la justificación de la calificación y la presentación explícita de los sistemas de calificación al principio de la asignatura.

La realización de procesos de evaluación formativa sigue siendo poco habitual en las aulas universitarias; situación especialmente preocupantemente en estos momentos de Convergencia hacia el EEES. Parece que se ha avanzado poco en 20 años y que el uso de procesos de evaluación formativa y continua siendo algo tan raro y poco habitual, tanto en la docencia universitaria como en la FIP.

En relación con la FIP los resultados son doblemente graves, puesto que parecen mostrar que se están realizando prácticas docentes considerablemente incoherentes con los discursos que se desarrollan en las asignaturas, así como con la legislación educativa española de los últimos 40 años. Existen otros trabajos que han denunciado las graves consecuencias de que en la FIP se reciban discursos sobre cómo hacer la evaluación en la práctica educativa que son completamente incoherentes con la evaluación que se lleva a cabo en dichas asignaturas (Fernández-Pérez, 1989; López-Pastor, 2009; Red IRES, 2010; Tejedor, 1998; Tonucci, 2010). Por ello, Fullan (1991) defiende que en la FIP es fundamental predicar con el ejemplo. Hamodi y López (2012), en un estudio cualitativo, encuentran que el alumnado de FIP muestra un consenso claro sobre que la evaluación formativa ayuda a aprender más y mejor y demanda una mayor coherencia entre el discurso pedagógico y la práctica educativa de sus profesores. En López-Pastor (2008) y Santos, Martínez y López-Pastor (2009) pueden encontrarse experiencias de innovación

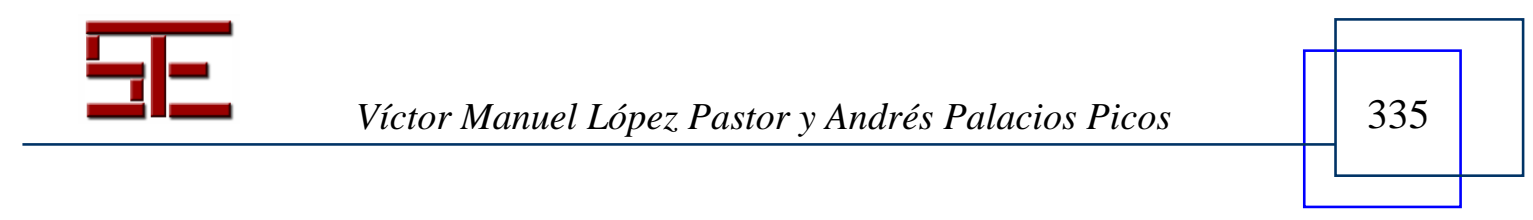




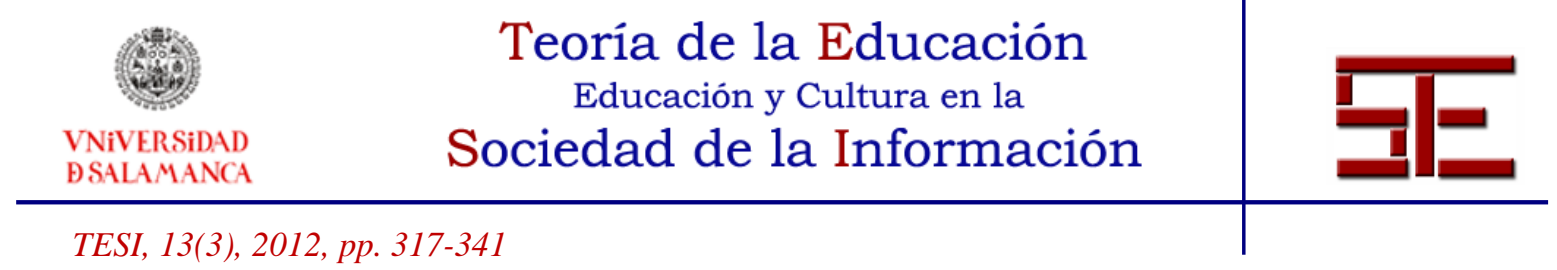

en las que el alumnado de FIP considera que el desarrollo de procesos de evaluación formativa en algunas asignaturas a lo largo de su formación inicial les ha ayudado en el desarrollo de sus competencias profesionales.

Este escaso desarrollo de una EOA se hace más evidente en la FIP de secundaria. Las diferencias en las percepciones entre ambos tipo de alumnado (futuros maestros de primaria vs futuros profesores de secundaria) son evidentes e indicadoras de un mayor compromiso con sistemas de EOA de los docentes de las diplomaturas, así como una prevalencia de modelos clásicos de evaluación en la licenciatura.

En otro orden de cosas, la presencia de altas correlaciones en los diferentes aspectos analizados de la evaluación y de la calificación podría enmascarar lo dicho en los párrafos anteriores sobre un aumento progresivo en los porcentajes de docentes comprometidos con sistemas de EAO. Hemos encontrado altas correlaciones entre autoevaluación, coevaluación, autocalificación y calificación dialogada; las hemos vuelto a encontrar entre la realización de procesos de negociación curricular sobre el sistema de evaluación, la explicación previa de los criterios de evaluación y calificación y la evaluación de los programas de las asignaturas; así como entre consensuar los criterios de evaluación, evaluar el programa de la asignatura y hacer explícitos los criterios de evaluación. Podría pensarse que estas altas correlaciones indican la existencia de pocas asignaturas con estilos de EOA, en las que se utilizan estrategias variadas que hacen que aumenten los porcentajes generales de uso, dando la falsa imagen de una mayor utilización de lo que realmente se usa.

Como conclusión, en este trabajo hemos presentado la valoración que el alumnado realiza sobre las prácticas de evaluación que se llevan a cabo en la FIP. Dichos resultados muestran que continúan predominando los modelos tradicionales de docencia universitaria, que hemos denominado Evaluación Orientada a la Calificación (EOC), frente a modelos innovadores de evaluación formativa, denominados Evaluación Orientada al Aprendizaje (EOA). Las prácticas de EOA son todavía minoritarias en la FIP, lo cual es preocupante en este tipo de estudios y, especialmente, en la actual situación de convergencia hacía el EEES. No obstante, los datos muestran también que se pudiera estar avanzando respecto a estudios realizados hace una y dos décadas. Así mismo, parecen generalizarse prácticas en línea con sistemas de EOA, como la diversificación de instrumentos de calificación y la explicitación de los criterios de evaluación y calificación. En cambio, son preocupantes las numerosas diferencias estadísticamente significativas que parecen existir entre la FIP de primaria y de

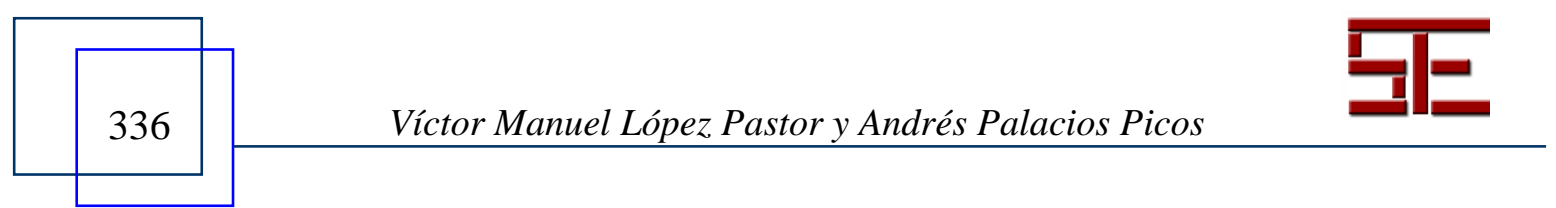




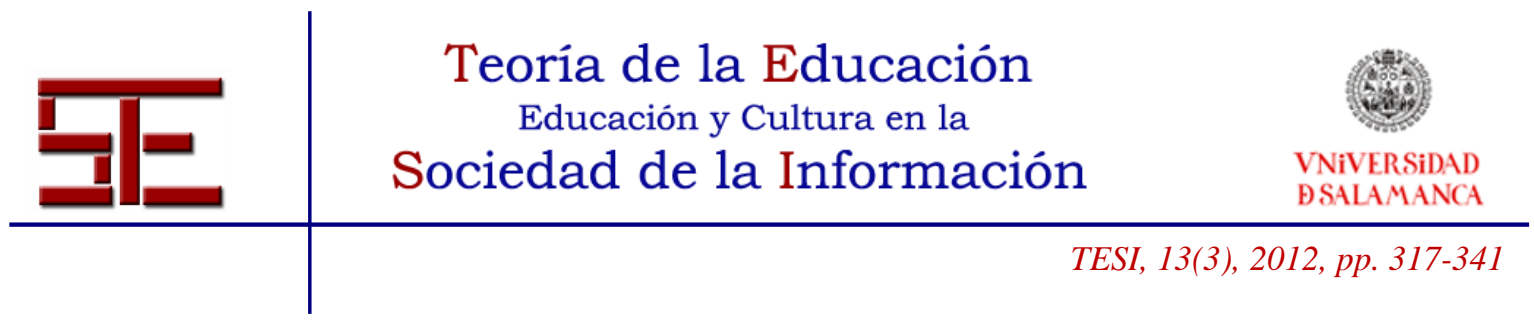

secundaria, dado que en esta última la existencia de asignaturas con una EOA parece ser mucho más escasa y minoritaria.

No obstante el panorama poco alentador de los sistemas de evaluación en las aulas universitarias, la literatura internacional especializada lleva muchos años acumulando estudios y evidencias sobre la necesidad de utilizar la evaluación como estrategia para mejorar y favorecer los aprendizajes, y no como simple certificación del éxito o fracaso. En estos momentos pueden encontrarse abundantes estudios y experiencias que demuestran que es posible desarrollar sistemas de evaluación orientados al aprendizaje y que dichos sistemas tienen efectos positivos en el aprendizaje del alumnado.

Para terminar, quisiéramos señalar algunos aspectos a mejorar en próximos trabajos y superar algunas limitaciones del actual. Nuestro estudio ha contado con un número importante de alumnos de las diplomaturas de Magisterio y de la Licenciatura de CCAFyD. Puede ser interesante explorar si las diferencias que hemos encontrado entre ambas titulaciones puedan ser mayores, o al menos diferentes, al ampliar la muestra a otros estudios y otras titulaciones. Por último, la utilización de datos cuantitativos, siendo una estrategia sólida y fiable, no permite afrontar la compleja realidad del mundo de las aulas en toda su dimensión. Se hace necesario atender un enfoque integral de la evaluación que incorpore una perspectiva cualitativa. Nuestra intención para futuras investigaciones es utilizar también métodos cualitativos de investigación, incorporando un análisis interpretativo, para profundizar en la percepción que tiene el alumnado sobre la evaluación recibida, lo que sin duda mejorará los resultados y conclusiones obtenidas.

\section{5.- BIBLIOGRAFÍA}

Biggs, J. (2005). Calidad del aprendizaje universitario. Madrid: Narcea.

Black, P. \& Williams, D. (1998). Assessment and Classroom Learning. Assessment in Education, 4 (1), 7-71.

Bonsón, M. y Benito, A. (2005). Evaluación y Aprendizaje. En A. Benito y A. Cruz (Eds.), Nuevas claves para la docencia universitaria en el Espacio Europeo de Educación Superior (pp. 87-100). Madrid: Narcea.

Boud, D. \& Falchikov, N. (2007). Rethinking Assessment in Higher Education. Learning for the long term. Oxon: Routledge.

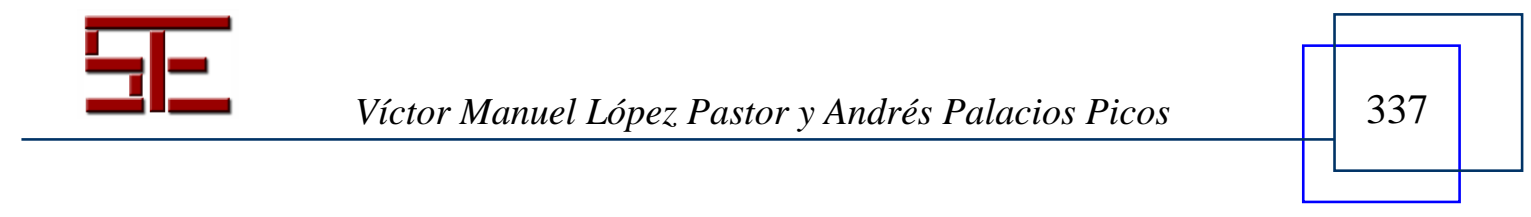




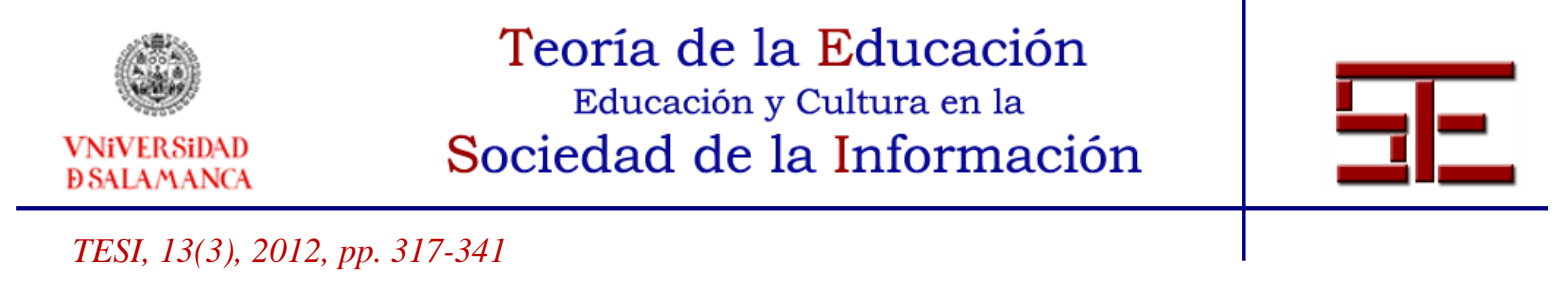

Brockbank, A. \& McGill, I. (2002). Aprendizaje reflexivo en la educación superior. Madrid: Morata.

Brown, S., \& Glasner, A. (2003). Evaluar en la universidad. Problemas y nuevos enfoques. Madrid: Narcea.

Carless, D., Joughin, G. \& Mok, M. M. C. (2006). Learning-oriented Assessment: Principles and Practice. Assessment \& Evaluation in Higher Education, 31 (4), 395-398.

Dochy, F., Segers, M. \& Dierick, S. (2002). Nuevas vías de aprendizaje y enseñanza y sus consecuencias: una era de evaluación. Red Estatal de Docencia Universitaria, 2 (2), 13-30.

Falchikov, N. (2005). Improving Assessment Through Student Involvement. Practical solutions for aiding learning in higher and further education. Oxon: Routledge.

Fernández-Balboa, J. M. (2006). Dignity and democracy in the college classroom: The practice of self-evaluation. In R. A. Goldstein, Useful Theory: Making Critical Education Practical. New York: Peter Lang Publishing.

Fernández-Pérez, M. (1989). Así enseña nuestra universidad. Salamanca: Hispagraphis. Flórez, J. (1999). Evaluación de la calidad de la docencia. León: Universidad de León.

Freire, P. (1990). La naturaleza política de la Educación: Cultura, Poder y Liberación. Barcelona: Paidós-MEC.

Fullan, M. (1991). The New Meaning of Educational Change. Cassell: Londres.

Gargallo, A. (2009). Aproximación al estudio de la evaluación en la universidad: un análisis exploratorio con alumnos semipresenciales. Contextos Educativos, 12, 77-93.

Gibbs, G. (2003). Uso estratégico de la evaluación en el aprendizaje. En S. G. Brown \& A. Glasner (Eds.), Evaluar en la universidad. Problemas y nuevos enfoques (pp. 61-75). Madrid: Narcea.

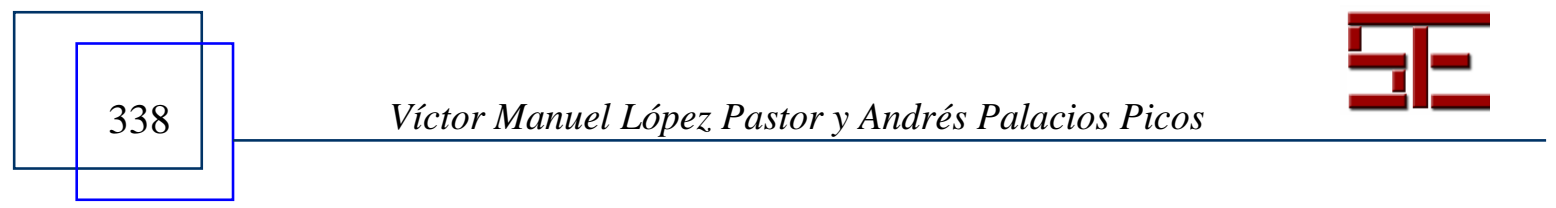




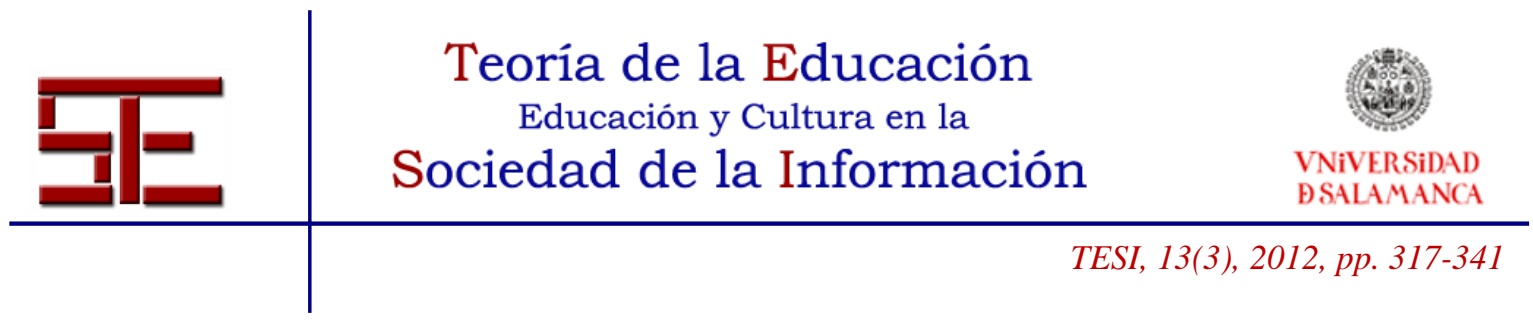

Gros, B. y Romaña, T. (1995). Ser profesor. Palabras sobre la docencia universitaria. Barcelona: Universidad de Barcelona.

Hamodi, C. y López, A. T. (2012). La evaluación formativa y compartida en la Formación Inicial del Profesorado desde la perspectiva del alumnado y de los egresados. Psychology, Society, \& Education, 4 (1), 99-112.

Ibarra, M. S., y Rodríguez-Gómez, G. (2010). Aproximación al discurso dominante sobre la evaluación del aprendizaje en la universidad. Revista de Educación, 351, 385407.

Knight, P. (2005). El profesorado de Educación Superior. Formación para la excelencia. Madrid: Narcea.

López-Pastor, V. M. (2008). Implementing a Formative and Shared Assessment System in Higher Education Teaching. European Journal of Teacher Education, 31 (3), 293 311.

- (coord.) (2009). La Evaluación Formativa y Compartida en Docencia Universitaria: propuestas, técnicas, instrumentos y experiencias. Madrid: Narcea.

Martínez, L., Martín, M. y Capllonch, M. (2009). Una experiencia de desarrollo profesional docente universitario de Educación Física a través de una práctica crítica, reflexiva y colaborativa. Cultura y Educación, 21 (1), 95-106.

McMahon, T. (2010). Peer feedback in a undergraduate programme: using action research to overcome students' reluctante to criticise. Educational Action Research, 18 (2), 273-288.

Nieto, S. (2000). El discurso del profesorado universitario sobre la evaluación del aprendizaje de los alumnos como estrategia de innovación y cambio profesional: exposición y análisis de una experiencia. Revista de Educación, 322, 305-324.

Pérez, P., Pozo, J. I. y Rodríguez, B. (2003). Concepciones de los estudiantes universitarios sobre el aprendizaje. En J. I. Pozo y C. Monereo, La universidad ante la nueva cultura educativa. Enseñar y aprender para la autonomía. Madrid: Síntesis$\mathrm{UAB}$.

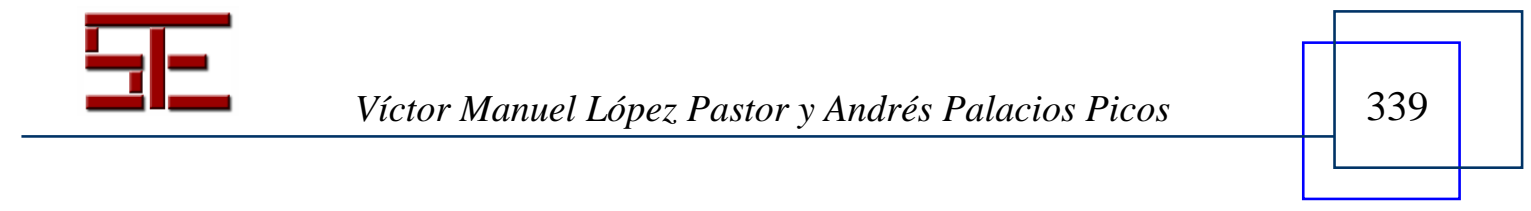




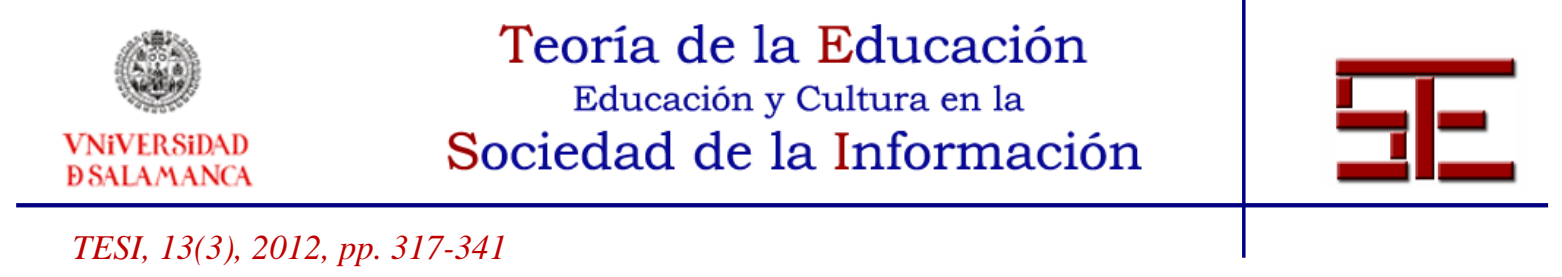

Red IRES (Investigación y Renovación escolar) (2010). No es verdad. Manifiesto pedagógico. Educar(nos), 49, 5-8.

Rodríguez-Gómez, G. (Coord.) (1998). La evaluación desde la perspectiva del alumnado universitario: Propuestas para la mejora. Revista de Enseñanza Universitaria (Número Extraordinario), 223-234.

Sanmartí, N. (2007). (10 ideas clave) Evaluar para aprender. Barcelona: Grao.

Santos, M., Martínez, L. F. y López, V. M. (coords.) (2009). La Innovación docente en el Espacio Europeo de Educación Superior. Almería: Universidad de Almería.

Shepard, L. A. (2000). The Role of Assessment in a Learning Culture. Educational Researcher, 29 (7), 4-14.

Tabernero, B., De las Heras, J. M. y González-Boto, R. (2009). Evaluación formativa y portafolios del estudiante. Tándem: Didáctica de la Educación Física, 31, 49-61.

Tejedor, F. J. (Dir.) (1998). Las estrategias utilizadas por los profesores universitarios para la evaluación del aprendizaje de los alumnos. Madrid: CIDE-Informe.

Tonucci, F. (2010). Apuntes sobre el manifiesto. Educar(nos), 49, 9-11.

Trillo, F. (2005). La evaluación de los estudiantes universitarios. El caso de la Universidad de Santiago de Compostela. Santiago de Compostela: Universidad de Santiago de Compostela.

Trillo, F. y Porto, M. (1999). La percepción de los estudiantes sobre su evaluación en la universidad. Un estudio en la Facultad de Ciencias de Educación. Revista de Innovación Educativa, 9, 55-75.

Zabalza, M. (2003). Diseño curricular en la universidad. Competencias del docente universitario. Madrid: Narcea.

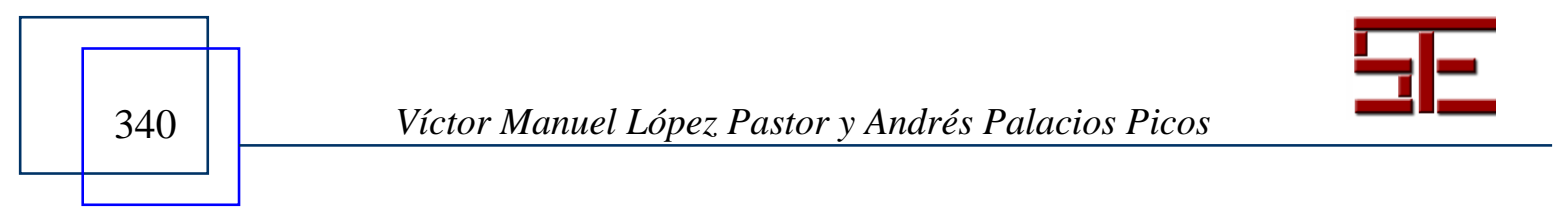




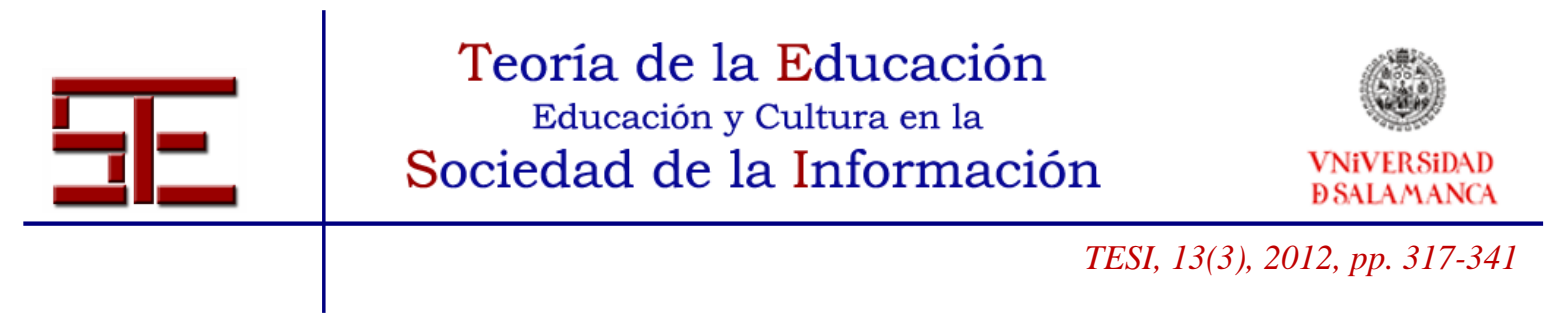

Para citar el presente artículo puede utilizar la siguiente referencia:

López Pastor, V. M. y Palacios Picos, A. (2012). Percepción de los futuros docentes sobre los sistemas de evaluación de sus aprendizajes. Revista Teoría de la Educación: Educación y Cultura en la Sociedad de la Información. 13(3), 317-340 [Fecha de consulta: dd/mm/aaaa].

http://campus.usal.es/ revistas_trabajo/index.php/revistatesi/article/view/9143/9376 